Michael Lehner

\title{
»Und nun sehe man, was hieraus gemacht werden kann«. \\ Carl Czernys Anleitung zum Fantasieren als implizite Harmonie- und Formenlehre
}

Will man der Improvisationskultur im Wien des frühen I9. Jahrhunderts auf die Spur kommen, ist Carl Czerny eine Schlüsselfigur: Er stellt nicht nur als Lehrer und Pianist eine Autorität auf diesem Gebiet dar, sondern beschäftigt sich auch in Lehrwerken und anderen Schriften mit dem »Fantasieren«. Darüber hinaus spiegelt sich das improvisatorische Moment in seinen Kompositionen wider, ein großer Teil der Klavierwerke verweist allein in der Gattungsbezeichnung und Titelwahl darauf: Fantasien (darunter insbesondere die zu seiner Zeit immer beliebter werdenden Opernfantasien), Variationen, Potpourris, Capricci, Präludien et cetera. ${ }^{\mathrm{I}}$

Trotz des in den letzten Jahren zunehmenden Interesses an der Person Carl Czernys $^{2}$ blieb dieser zentrale Aspekt seines Schaffens erstaunlicherweise fast gänzlich ausgespart. ${ }^{3}$ Jenseits einer zunehmenden Wertschätzung seiner Musik, die sich auch an vermehrter Tonträger-Produktion ablesen lässt, rückte zwar durchaus sein pädagogisches Schaffen in den Fokus der Forschung, jedoch vor allem im Hinblick auf Fragen zu Interpretationsforschung und historischer Aufführungspraxis: Seine Ausführungen zur Vortragskultur oder seine Bemerkungen zur Aufführung der Sonaten Beethovens liefern wertvolle Informationen und Details über die Klavierkultur des frühen I9. Jahrhunderts - seien es Bemerkungen allgemeiner Art über Sitz, Haltung oder Kleidung des Pianisten, die Position und Platzierung des Pianofortes bis hin zu genauen Angaben über Phrasierung, Artikulation, Tongebung, Anschlag, oder detaillierte Bemerkungen zur Ausführung und Interpretation einzelner Passagen der Klavierliteratur.

1 Vgl. dazu Michael Saffle: Czerny and the Keyboard Fantasy. Traditions, Innovations, Legacy, in: Bepond the Art of Finger Dexterity. Reassessing Carl Cerny, hg. von David Gramit, Rochester 2008, S. 202-229.

2 Symposien, Ausstellungen und Sammelbände versuchen Czernys Schaffen aus den festgefügten Bildern und Klischees zu befreien; vgl. etwa Carl Czerny. Komponist, Pianist, Pädagoge, hg. v. Heinz von Loesch, Mainz 2010 und »Mehr Respekt vor dem tüchtigen Mann«. Carl Czerny (I79I-I857), Komponist, Pianist, Pädagoge, hg. von Urs Fischer und Laurenz Lütteken, Kassel u. a. 2009.

3 Die Sammelbände von Fischer/Lütteken und von Loesch behandeln das Thema gar nicht, man ist auf die grundlegende Arbeit Grete Wehmeyers sowie auf Ulrich Mahlert verwiesen: Grete Wehmeyer: Carl Czerny und die Einzelhaft am Klavier oder Die Kunst der Fingerfertikeit und die industrielle Arbeitsideologie, Kassel u. a. I983, S. I35-150; Ulrich Mahlert: Vorwort, in: Carl Czerny: Spstematische Anleitung zum Fantasieren auf dem Pianoforte, op. 200, Wien [I829], hg. und mit einer Einl. vers. von Ulrich Mahlert, Wiesbaden u. a. I993, S. III-Xv. 
In diesem Beitrag hingegen wird der Versuch unternommen, Czernys zentrale Vorstellungen einer guten und angemessenen Improvisationskunst herauszuarbeiten und im Kontext der historischen Situation der Beethoven-Ära zu verorten. Hierbei wird deutlich, wie stark schriftliches Komponieren mit Improvisation in Wechselwirkung steht. Zwar haben Gattungsmerkmale schriftlich fixierter Kompositionen einen großen Einfluss auf improvisatorische Strukturen, den Czerny selbst ausführlich beschreibt. Es kann aber ebenso gezeigt werden, dass der Einfluss der Improvisationspraxis auf das Entstehen und die Entwicklung formaler Aspekte des Komponierens noch immer unterschätzt wird. Dies wird am Beispiel der »double function form« verdeutlicht werden.

Die Systematische Anleitung zum Fantasieren auf dem Pianoforte als Schlüsselwerk Czernys umfangreiche Fantasierschule von I829, die zwei Jahre nach Beethovens Tod als Opus 200 erschien, ist gleichsam ein Kompendium improvisatorischer Stile; in Anspruch und Vollständigkeit überragt es alle anderen pädagogischen Schriften zum Thema. Gewissermaßen abschließend stellt es die gebräuchlichen improvisatorischen Gattungen und Techniken der Beethoven-Zeit zusammen. Viele davon werden bereits wenige Jahre später ihre Bedeutung für die Ausbildung und die Konzerttätigkeit der Pianistengeneration der zweiten Hälfte des I9. Jahrhunderts verlieren. Auch Czernys eigene Klavierschule op. 500 aus dem Jahr I839 lässt bereits erkennen, wie sehr das freie Spiel, zumindest für das öffentliche Konzertwesen, an Bedeutung verliert.

Die wenigen existierenden Forschungsbeiträge zu Czernys Improvisationsverständnis gehen vornehmlich auf seine kurzen schriftlichen Kommentare zu Improvisation und Fantasie in den Lehrwerken ein - insbesondere jene aus op. 200. Die Modellkompositionen selbst werden indes so gut wie nie als eigenständige Quelle behandelt, dabei stellen gerade diese Beispiele den eigentlichen Ansatz Czernys in Notenform dar. ${ }^{4}$ Neben kurzen einleitenden Ausführungen zu Beginn der Kapitel sowie einem kurzen Vor- und Nachwort besteht die Fantasierschule fast ausschließlich aus Notenbeispielen, seine Präludierschule op.30o kommt sogar gänzlich ohne Beschreibungen und Erklärungen aus. Obwohl Czerny sein Lehrwerk als »systematische Anleitung « begreift, gibt er so gut wie keine Hinweise zum methodischen Erlernen dieser Kunstfertigkeit - man muss seinen pädagogischen Ansatz gleichermaßen aus den Notenbeispielen herausdestillieren. In ihnen stecken die Möglichkeiten der harmonischen und formalen Gestaltung, der Melodiebildung, Themenaufstellung und -verarbeitung, der unterschiedlichen Satzstrukturen, der charakteristische Umgang mit Wiederholung, Variation und Kontrast

4 Dies stellt bereits Lutz Felbick fest; vgl. ders.: Vom Einfluss der Improvisation auf das mitteleuropäische Musikleben des I9. Jahrhunderts, in: Musiktheorie 20 (2005), H. 2, S. I68-I82, insb. S. 173 . 
verborgen - wenngleich im Notierten das Unmittelbare des Stegreifspiels nur noch als ein »als ob « gefasst werden kann.

Neben Czernys Lehrwerken, die Improvisation behandeln (neben op. 200 auch die Präludierschule op. 300 , die Klavierschule op. 500 und die Briefe über den Unterricht aufdem Pianoforte vom Anfange bis zur Ausbildung5), eignen sich seine Kompositionen, so die These, mehr als andere, um Gestalt und typische Muster improvisierter Musik der Zeit zu rekonstruieren, da sein Kompositionsprozess zunächst generell einer ist, der - gleichsam wie beim Improvisieren selbst - über eine Vielzahl an Bausteinen, Floskeln und am Klavier eingeübter und improvisierter Wendungen zu einem Werkganzen gerät, ${ }^{6}$ das als aufgeschriebene Komposition stets seine Nähe zum Haptischen, am Klavier gefundenen bewahrt. Bei Stücken, die von ihrer Gattungsgeschichte her generell eine Nähe zum Improvisieren aufzeigen, gilt dies freilich umso mehr.

Die Modellkompositionen in op. 200 unterscheiden sich jedoch auch von seinen eigenen komponierten Fantasien. So reflektiert etwa die Fantasie op. 27 sowohl Gattungstraditionen als auch die Beispiele berühmter Vorläufer, wie etwa Beethovens Fantasie op. 77. Sie bildet damit eine Brücke zwischen einer zwar aus Improvisation abgeleiteten, jedoch hinsichtlich der Komplexität von Strukturen und der Verarbeitung von Themen sorgfältig komponierten Musik. Das bewusst Unfertige seiner Modellstücke thematisiert Czerny dabei explizit:

"Indem ich hier einige Beyspiele nachfolgen lasse, kann es nicht meine Absicht seyn, vollkommen durchgeführte Muster aufstellen zu wollen. Nur die Grundrisse, nur der beyläufige Gang der Ideen, lassen sich hier schriftlich darlegen, und als das, kann es dem Schüler immer ein Leitfaden seyn. Ich zeige hier und da an, wo eine verlängerte Ausführung nöthig wäre, worin sich auch der Schüler zu versuchen hat.«?

In den Beispielen selbst liegt also Czernys eigene Idealvorstellung von gelungener Improvisation verborgen. Man könnte sie demnach als Quasi-Improvisationen begreifen, die versuchen, die Flüchtigkeit improvisierter Musik auch im Notentext zu bewahren Ulrich Mahlert charakterisiert sie in diesem Sinne treffend als »zensurierte Fantasie-

5 Carl Czerny: Die Kunst des Präludierens in r2o Beispielen, Präludien, Modulationen, Cadenzen, Fantasien aller Gattungen, Wien I833; ders.: Vollständige theoretisch-practische Pianoforte-Schule, op.500, Dritter Teil: Von dem Vortrage, Wien I839, Nachdruck hg. und mit einer Einl. vers. von Ulrich Mahlert, Wiesbaden I99I; ders.: Briefe über den Unterricht auf dem Pianoforte vom Anfange bis zur Ausbildung; als Anhang zu jeder Clavierschule, Wien [ca. I839/40]. Das Erscheinungsjahr ist nicht vermerkt, Czerny spricht aber im Vorwort an, dass die Briefe kurz nach der Klavierschule von I839 entstanden seien, um in Briefform Schüler »von Schritt zu Schritt« zu leiten, also eine gewisse Nähe zum realen Klavierunterricht zu erreichen.

6 Vgl. hierzu auch Wehmeyer: Carl Czerny, S. 69-75. Nur durch diese ökonomische Arbeitsweise lässt sich auch das riesige Werkkorpus erklären.

7 Czerny: Systematische Anleitung zum Fantasieren, S. 44 
protokolle «. ${ }^{8}$ Bevor jedoch näher auf ihre Ausgestaltung eingegangen wird, soll der Stellenwert der Improvisation in Czernys Schaffen kurz skizziert werden.

Zum Stellenwert der Improvisation im đuvre Czernys Mehrere Lehrwerke und Übungsstücke Czernys widmen sich der Improvisation, bezeichnenderweise ist der Großteil der frühen Veröffentlichungen der I82oer- und 3oer-Jahre dem Thema gewidmet. Darunter befinden sich auch die ersten beiden theoretischen beziehungsweise pädagogischen Lehrwerke zum Thema überhaupt, die Fantasierschule op. 200 und die Präludierschule op.300, ebenso ein großer Teil der frühen Übungen und Studien wie die Sammlungen op. 6I und I6I.

Op. 200 ist das erste größere systematische Lehrwerk Czernys; seine große Klavierschule op. 500 und die bedeutenden, bis heute bekannten und verwendeten Studienwerke für Klavier (Schule der Geläufigkeit op. 299, I60 kurze Übungen op. 82I et cetera) erschienen allesamt erst, als er längst nicht mehr unterrichtete. ${ }^{9}$

In den späteren Jahren verschiebt sich der Fokus zu den bekannten Themen wie Geläufigkeit der Finger, technische Souveränität und pianistische Virtuosität. Tabelle I zeigt alle Lehrwerke und Sammlungen zum Thema. ${ }^{\text {Io }}$

op.6I Präludien, Cadenzen, und kleine Fantasien

op. I6I 48 Etudes en forme de Préludes et Cadences dans tous les tons [...]

op. $200 \quad$ Systematische Anleitung zum Fantasieren

op.300 Die Kunst des Präludierens

op.3I5 Cadenzen zu Ludwig van Beethovens Concerten, 2 Bde.

op.355 Die Schule der Verzierungen, Vorschläge, Mordenten und Triller [...]

op.500 Vollständig theoretisch-practische Pianoforte-Schule

o. op. Briefe über den Unterricht auf dem Pianoforte

TA B ELLE 1 Sammlungen und Lehrwerke Czernys zum Thema Improvisation

8 Mahlert: Einführung, in: Czerny: Systematische Anleitung zum Fantasieren, S. viII.

9 Davor hat er existierende Klavierschulen überarbeitet und aktualisiert: August Eberhards Müllers Klavier- und Fortepiano-Schule (I804) als Grosse Fortepiano-Schule (I825) sowie Ignaz Joseph Pleyels Méthode pour piano-forte par Plepel et Dussek (I797) als Plepel's Clavierschule (1826); vgl. Wehmeyer, Art: »Czerny, Carl«, in: MGG2, Personenteil, Bd. 5, Kassel u. a. 200I, Sp. 220-233, hier Sp. 225. Vgl. Werkverzeichnis in: Carl Czernp. Erinnerungen aus meinem Leben, hg. und mit Anm. vers. von Walter Kolneder, Straßburg/Baden-Baden I968, S. 55-76. Einzelpublikationen von Fantasien, Capricci et cetera wurde nicht berücksichtigt. Ein aktuelles, kritisches Werkverzeichnis Czernys liegt bis auf den heutigen Tag nicht vor. Die existierenden Verzeichnisse von Pazdírek und Kolneder weichen teilweise voneinander ab; vgl. Universal-Handbuch der Musikliteratur aller Zeiten und Völker, hg. von Franz Pazdírek, Wien I904-I9ıo, Bd. 6, S. 66I-688. 
Darüber hinaus ändert sich die Stellung der Improvisation über die Jahrzehnte hinweg: So wird sie in der Klavierschule op. 500 beispielsweise nicht mehr als »besondere Pflicht und Zierde « ${ }^{\text {II }}$ bezeichnet, sondern als »höchst interessante und ehrenvolle Kunst «, ${ }^{\text {I2 }}$ die nur dem »wahren Virtuosen« abverlangt werden kann, wenn auch bereits eingeschränkt. In dieser Definition des Virtuosen ist ein besonders ausgeprägtes Improvisationstalent nicht mehr unbedingte Bedingung: »Daher wird ein wahrer Virtuose stets, wenigstens bis zu einem gewissen Grade, zu fantasieren im Stande sein, selbst wenn ihm zu dieser Kunst ein bestimmtes Talent mangeln sollte.«33

Die gegebenen Beispiele zum Präludieren in op.500 sind nur noch kurz und die Aufgaben des Präludierens stärker eingeschränkt, ebenso die improvisatorischen Freiheiten im Vortrag fremder Stücke, die nur noch beim brillanten Repertoire statthaft sind.

Czerny ließ in der Bearbeitung der Klavierschule von August Eberhard Müller I825 noch zu, dass man eine »melodische Stelle zur rechten Zeit« bei entsprechender »Kenntniß der Regeln der Harmonie « »auf mancherley Weise verändern könne«. ${ }^{14}$ Nur vier Jahre später differenziert er im dritten Kapitel seines op. 200 »Von den Cadenzen, Fermaten und längeren Verzierungen«:

»In Werken von tiefen [sic] Gehalt und ernsten Charakter (z. B. Beethovens Sonate, D mol, Op. 29) wäre jede Art von Zugabe sehr übel angewendet. Dagegen in Compositionen, die vorzugsweise für ein glänzendes, delikates oder sentimentales Spiel berechnet sind, in Variationen, Potpourris, arrangirten Gesangsstücken, oder sonstigen Produkten des herrschenden Geschmacks, giebt es häufige Gelegenheiten, wo dergleichen kleine Impromptus angemessen, ja oft Bedürfniss sind, um eine vielleicht sonst kahle und schleppende Stelle auszuschmücken. ${ }^{\mathrm{I} 5}$

Czerny befindet sich damit in einer Zwischenposition: Bei bereits kanonisierten Werken, insbesondere dem Werk Beethovens, sind Zusätze unstatthaft. Im brillanten oder sentimentalen Genre, das den Massengeschmack der Zeit widerspiegelt, sind sie indes durchaus erwünscht, ähnlich wie zusätzliche Präludien ihm hier angemessen scheinen. »Dass zu ernsteren Werken (z. B. zu Beethovens Sonate F mol, op. 57 u. drgl) überhaupt keine solche längere Vorspiele anwendbar wären, versteht sich von selbst « ${ }^{\mathrm{I} 6}$ auch wenn $\mathrm{ihm}$ wenige Takte immerhin denkbar scheinen. Die längeren Präludien sind der improvisatorischen Vorbereitung von Rondos, Variationen und anderen »brillanten« Bravour-

Ders.: Vollständige theoretisch-practische Pianoforte-Schule, Bd.3, S. 9I.

Ebd.

August Eberhard Müller: Grosse Pianoforte-Schule, [...], mit vielen neuen Bepspielen und einem vollständigen Anhange vom Generalbass, versehen von Carl Czerny, Leipzig [1825], S. 234.

Czerny: Systematische Anleitung zum Fantasieren, S. 22. Vgl. dazu auch Wehmeyer: Carl Czernp, S. 149 .

16 Czerny: Systematische Anleitung zum Fantasieren, S. I5. 
stücken vorbehalten - doch auch diese Gattung verschwindet in seinen späteren Lehrwerken vollständig.

In seinen Ausführungen »Über den richtigen Vortrag der sämmtlichen Beethovenschen Werke für das Piano allein«geht Czerny dazu über, das gesamte Beethoven-CEuvre für sakrosankt, und Veränderungen und Vorspiele für unstatthaft zu erklären. Unmissverständlich lässt er die Passage gesperrt und mit vergrößertem Zeilenabstand setzen: »Beim Vortrage seiner Werke, (und überhaupt bei allen klassischen Autoren) darf der Spieler sich durchaus keine Änderung der Composition, keinen Zusatz, keine Abkürzung erlauben. «77

Binnen guter zehn Jahre wird die Bedeutung des Improvisierens marginalisiert: Das einleitende Präludieren wird immer unwichtiger, sämtliche Hinzufügungen nicht nur bei Beethoven, sondern bei »allen klassischen Autoren« werden unstatthaft, eine Sichtweise, wie sie beispielsweise später in der Klavierschule von Moscheles nachzuvollziehen ist. $^{\text {I8 }}$

Im Gegensatz zu seinen eigenen Bemerkungen lässt sich Czernys Fantasierschule so weniger als das erste, sondern vielmehr als eines der letzten Lehrwerke zum Thema verstehen, das von der Selbstverständlichkeit von Improvisation als einer Grundbedingung für Klaviervirtuosen ausgeht. In seinem kompendienhaften Anspruch auf Vollständigkeit bildet es einen abschließenden Überblick über die Interpretationskultur seiner Zeit und reicht vom Vorbild Beethoven, der mehrfach Erwähnung findet, zurück bis zu Carl Philipp Emanuel Bach. Einzelne Abschnitte gehen zudem zurück bis zur kontrapunktischen Improvisationskultur der Orgeltradition »im gebundenen Stil - die einzige, die bis auf den heutigen Tag insbesondere in Form des improvisierten liturgischen Orgelspiels nicht nur unter Spezialisten, sondern als allgemein verbreitete Praxis Bestand hat. Mit dem Zeitgeist auf Augenhöhe, verlagern sich Czernys theoretische Schriften und Lehrwerke von der Improvisation zum Vortrag unveränderlicher Meisterwerke und zur angemessen technischen Ausbildung für diese Aufgabe in einer entstehenden Repertoire-Kultur.

Aufbau und Konzept von op. 200 Czerny behauptet, sein Lehrwerk in einer "progressiven Ordnung« gegliedert zu haben, »welche zur Verständlichkeit und zu schnellen

17 Ders.: Vollständige theoretisch-practische Pianoforte-Schule, Bd. 4, S. 34.

18 Vgl. dazu Wehmeyer: Carl Czernp, S. I48f. Einzig das Präludieren in Form von kurzen ausgeschmückten Kadenzen vor und zwischen Werken bleibt bei einigen Pianisten teilweise bis ins 20. Jahrhundert bedeutsam; vgl. Claudio Bacciagaluppi: Die Kunst des Präludierens, in: Zwischen schöpferischer Individualität und künstlerischer Selbstverleugnung. Zur musikalischen Aufführungspraxis im r9. Jahrhundert, hg. von Claudio Bacciagaluppi, Roman Brotbeck und Anselm Gerhard, Schliengen 2009 (Musikforschung der Hochschule der Künste Bern, Bd. 2), S. I69-I88. 
Fortschritten sich als die angemessenste zeigt. « ${ }^{\mathrm{I} 9}$ Tatsächlich trifft dies jedoch nur auf den Beginn der Schrift zu. Die kleinen und länger ausgeführten Präludien vor dem Vortrag einer größeren Komposition lassen sich in der Tat bei sicherem Generalbassspiel, der Kenntnis und Ausführung von Modulationsverläufen sowie Versiertheit im Skalen- und Arpeggienspiel erlernen, insofern stellen die Präludien für Czerny eine Vorstufe dar. Für das 3. Kapitel »Von den Cadenzen, Fermaten und längeren Verzierungen« gilt dies zunächst ebenso: Die Ausgestaltungen der Fermaten sind nur reines Passagenwerk der rechten Hand, brillante Virtuosität steht im Vordergrund. Doch integriert Czerny hier bereits vollständige Kadenzen zu Klavierkonzerten und stellt als Exempel eine eigene Kadenz zu Beethovens erstem Klavierkonzert vor. Zwar handle es sich hierbei eigentlich schon um »selbstständige Fantasien«, trotzdem verlangt Czerny, im Sinne dieses Beispiels »zu allen Concerten dieser Art, solche Fermaten improvisieren« zu lernen. ${ }^{20}$ Damit unterwandert er den systematischen Aufbau in aufsteigendem Schwierigkeitsgrad und zwingt den Lernenden gewissermaßen, diese Passage zu überblättern, denn man kann dies unmöglich ausschließlich aus dem Wissen der vorangehenden Kapitel heraus leisten.

Zudem gewinnt seine Gattungssystematik zunehmend an Bedeutung, wie sich in den folgenden Kapiteln zeigt. So stellt das vierte Kapitel als eigentlich »erste Gattung des Fantasierens« das Improvisieren selbständiger größerer Tonstücke, die Fantasien über ein einzelnes Thema vor, das fünfte Kapitel die »2te Gattung«, Fantasien »über mehrere Themas «. Das sechste Kapitel spricht von der dritten Gattung, dem »Potpourri«, das sich von den vorigen durch die Verwendung bekannter Melodien, zumeist aus Opern, unterscheidet. Obwohl er zugibt, dass die erste Gattung eigentlich »die schwerste sei «, ${ }^{2 \mathrm{I}}$ stellt er sie trotzdem, dem eigenen Gattungsaufbau folgend, an den Beginn und gibt an dieser größten improvisatorischen Herausforderung erstmals Bemerkungen zur formalen Gestaltung sowie zur Themenentwicklung und -verarbeitung.

Ebenso erstaunt die weitere Einteilung: Die Kapitel 7, 8 und 9 widmen sich den Variationen (»4te Gattung «), dann dem »gebundenen und fugirten Styl (»5te Gattung«) und dem Capriccio (»6te Gattung«). Sie folgen dabei weder einem didaktischen Ansatz noch der begonnenen Gattungssystematik mit ansteigender Themenzahl: Kapitel 7 und 8 verhandeln improvisatorische Verläufe über ein Thema, Kapitel 9 geht schließlich zur maximalen Öffnung einer Vielfalt an Themen, Einfällen und Satzstrukturen in der Form des Capriccios über. Aus didaktischer Perspektive wäre es schlüssiger gewesen, die Va- 
riationen früher zu vermitteln, ${ }^{22}$ idealerweise nach den Präludien. Ohne umfangreiche Repertoirekenntnis und fortgeschrittene Selbständigkeit des Schülers lässt sich aus dem kurzen achten Kapitel das kontrapunktische Spiel nicht erlernen, es scheint eher der Vollständigkeit halber hinzugefügt worden zu sein. Bezeichnenderweise ändert Czerny etwa zehn Jahre später die Reihenfolge in den Briefen über die musikalische Erziehung. Seiner fiktiven Schülerin Cäcilie zählt er die gebräuchlichen Formen in anderer Reihenfolge auf und betont zudem sowohl einen sehr viel sinnvolleren aufsteigenden Schwierigkeitsgrad als auch für das i9. Jahrhundert relevantere Spielarten. So waren Variationen und Potpourris gerade in der Form der Opernfantasien in der Gunst des Publikums weit nach oben gerückt:

»Sie werden finden, dass fast alle in der Composition gebräuchlichen Formen auch im Fantasieren anwendbar sind. Man kann Variationen auf ein selbstgewähltes oder auch aufgegebenes Thema improvisieren. Man kann sehr interessante Pot-Pourris aus mehreren beliebten Motiven zusammenstellen, und durch brillante Passagen zu einem glänzenden Tonstück verbinden. Man kann sogar im strengern vierstimmigen Satz, oder im fugirten Style sich improvisierend auszeichnen u. s. w. « ${ }^{23}$

Fugenimprovisation wird zwar noch erwähnt, wie generell konzediert wird, dass alle Formen und Gattungen auch Teil improvisierenden Spiels sein können. Czernys Hauptgattungen aus op. 200 - das Fantasieren über ein oder mehrere spontan erfundene Themen sowie das Capriccio als Erbe der »freyen« Fantasie Carl Philipp Emanuel Bachs finden jedoch keine Erwähnung mehr.

In op. 200 definiert Czerny das Capriccio als Sonderfall einer Fantasie über mehrere Themen, die im Gegensatz zur vorgestellten »2ten Gattung« von größerer Freiheit geprägt sei, insbesondere durch »willkührliches Aneinanderreihen«, fehlende »besondere Durchführung « und ein »launiges schnelles Abspringen von einem Motiv zum andern, ohne weiteren Zusammenhang, als den der Zufall, oder, absichtlos, der Musiksinn des Spielers giebt. « ${ }^{24}$ Damit ist ein ursprünglich fester Bestandteil der Improvisationskultur des späten I8. Jahrhunderts an den Rand gedrängt. Von einer Improvisation größerer Tonstücke wird bei Czerny eine Nähe zu kompositorischen Strukturen der Zeit selbstverständlich erwartet. Der freie, auf Diskontinuitäten und Überraschungen beruhende Formverlaufbleibt nur als Ausnahme bestehen. Czerny räumt gleichwohl ein, dass eine Vielzahl von veröffentlichten Capricci dieser Definition nicht folgen und folglich der Begriff nicht wirklich trennscharf vom Terminus »Fantasie« gelöst werden kann. In der

Dies stellt bereits G. W. Fink in seiner Rezension zu Czernys Lehrwerk in der Allgemeinen musikalischen Zeitung fest; vgl. Mahlert: Einführung, in: Czerny: Systematische Anleitung zum Fantasieren, S. IX. 
Tat ist die Verwendung des Begriffes völlig uneinheitlich, ${ }^{25}$ scheint aber als Inbegriff besonders freier Passagen zumindest in Süddeutschland und Österreich bereits etabliert zu sein. Vielfach findet sich in Fantasien wie Sonaten Czernys und Hummels ebenso wie in zahlreichen Paraphrasen Liszts die Bezeichnung »a capriccio« für besondere metrische und rhythmische Freiheiten des Interpreten.

Präludien als angewandte Harmonielehre Czernys erstes Kapitel lässt sich als die praktische Ausführung einer »angewandten Harmonielehre« verstehen. Einzig in diesem Kapitel geht er partiell anleitend vor, indem er es nach Wissensstufen fortschreitend strukturiert, auch wenn sichere Beherrschung von Läufen und Passagen sowie ein ausgebildetes Avista-Spiel vorausgesetzt werden. Er verlangt hier nicht die Fähigkeit, Melodieverläufe und feste Satzstrukturen, Themen und Motive zu erfinden, sondern beschränkt sich auf die Ausgestaltung harmonischer Verläufe über Akkordfiguren und Passagenwerk - eine Wurzel des Präludierens, die zurück zu den Präludien, Toccaten und Intonazioni des Frühbarocks reicht. Einige der Modellpräludien lassen sich in diesem Sinne denn auch als frühromantische Neuschöpfung eines barocken Klangflächenpräludiums in der Improvisationstradition auf der Orgel verstehen.

Czerny stellt in op. 200 zunächst einfache Kadenzverläufe vor, die sämtliche gängigen prädominantischen Akkorde einführen. Die zweite, vierte und sechste Skalenstufe, auch deren Alternativen \#(4) und b(6) werden in Beispielen mit den typischen Akkordtypen vorgestellt. Sämtliche Beispiele sind komplett ausnotiert, Notenbeispiel I zeigt sie zusammengestellt in Generalbass-Reduktion.

Diese Kadenzen, wie etwa der »sehr gewöhnliche [...] Gang« (Notenbeispiel 2, a.I), werden nun ausgestaltet und erweitert. Czerny zeigt unterschiedliche Möglichkeiten der Erweiterung der Bassführung durch skalare Zwischenschritte, etwaige zusätzliche Harmonisierungen und insbesondere die Möglichkeiten der figurativen Ausgestaltung derselben, vornehmlich durch Lauf- und Akkordfiguren der rechten Hand. Der übergeordnete Kadenzverlaufbleibt indes derselbe. Dabei werden auch zusätzliche Sequenzen und chromatische Bassbewegungen in die Kadenzverläufe eingebaut (Notenbeispiel 4a).

Ab 15 geht Czerny einen Schritt weiter und verlässt den gefestigten Kadenzgrund. Es folgen Beispiele mit chromatischen und enharmonischen Akkordverbindungen, zumeist modulatorische Prozesse. Dabei spricht Czerny die Möglichkeit an, diese komplexeren Passagen auch in einer anderen Tonart als in jener des Beginns enden zu lassen. Hierin besteht eine historisch wichtige Funktion des Präludierens in der aufkommenden 

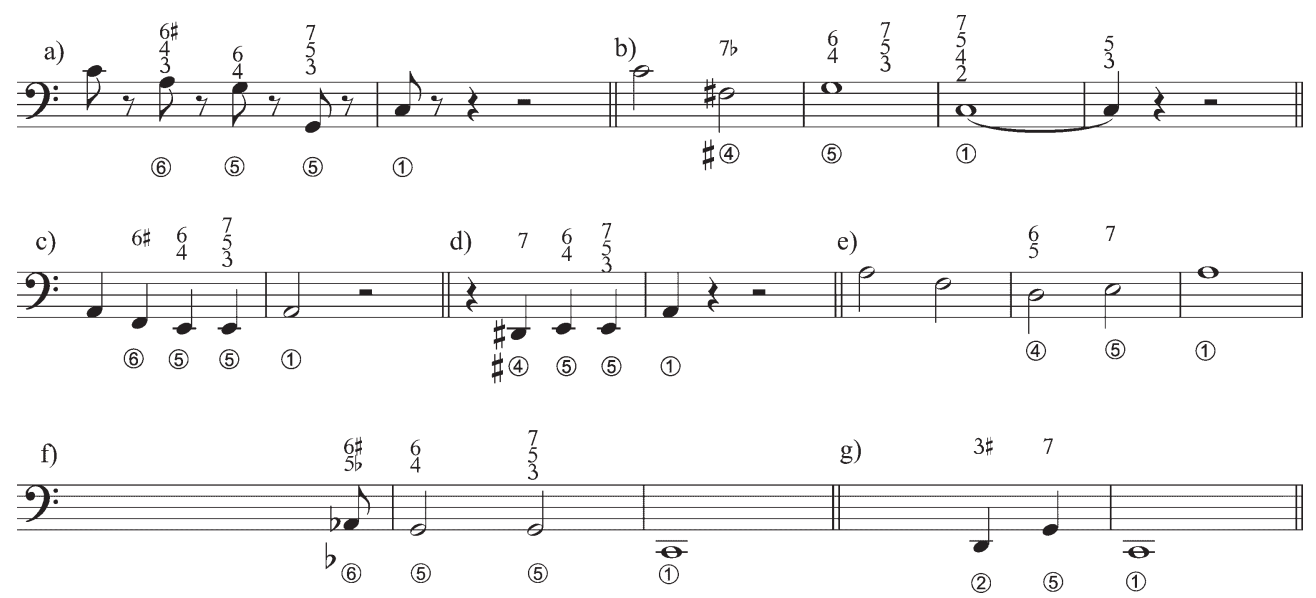

NOTENBEISPIEL 1 Akkordbildungen in einfachen Kadenzen in Czernys op. 200 (Reduktion) a) - e) J $_{3}$, S. 5; f) Exempel 5 (Schluss), S. 7; g) Exempel 12 (Schluss), S. 10
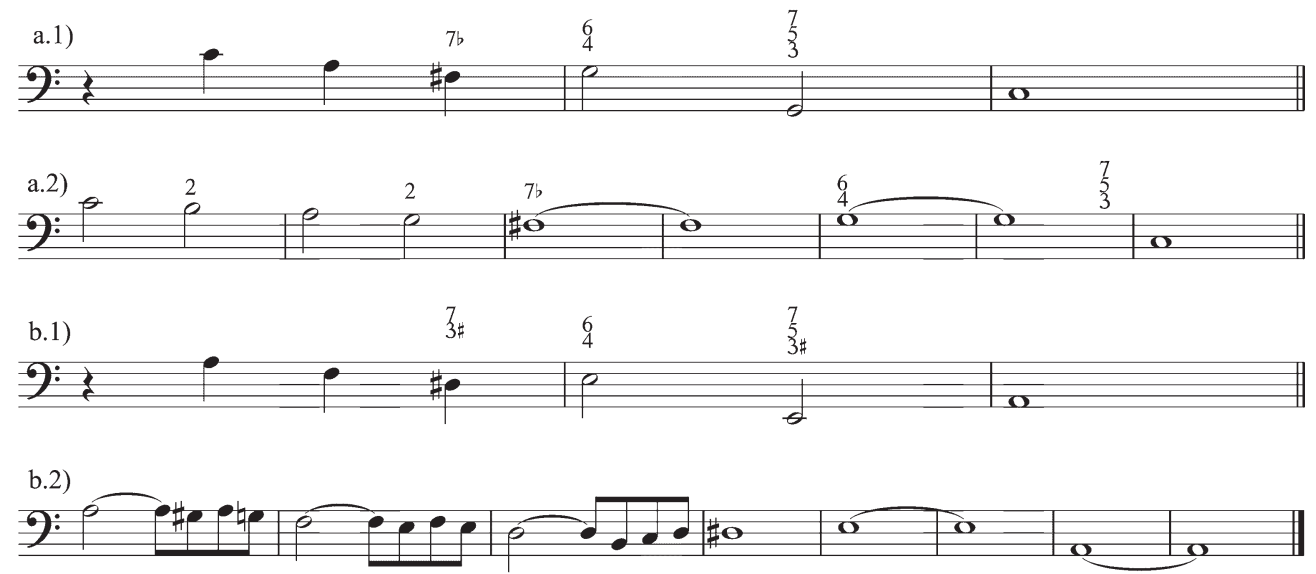

Noten BeISPIEL 2 Erweiterung der Bassverläufe einfacher Kadenzen

a.1) Exempel 1, S.6; a.2) Exempel 3, S.6; b.1) Exempel 7, S.8; b.2) Exempel 11, S. 9

Recital-Kultur des I9. Jahrhunderts: Durch die Annäherungen der Tonarten konnte eine Verbindung zwischen den (eigenen oder fremden) Stücken eines Klavierabends geschaffen werden. ${ }^{26}$ Interessanterweise erwähnt Czerny dieses vermittelnde Verfahren jedoch nirgendwo und spricht nur von der vorbereitenden Funktion. Da er jedoch bei klassischem Repertoire, insbesondere dem Werk Beethovens, längeres Präludieren ohnehin für unstatthaft erklärt und dies nur im »brillanten« Genre zulässt, scheint sich diese Funktion für ihn bereits erübrigt zu haben.

26 Vgl. Bacciagaluppi: Die Kunst des Präludierens, S. I73. 
Czerny geht in diesen Beispielen bis an die Grenzen der Tonalitätsvorstellung seiner Zeit und spricht von »kühne[n], fremdartige[n] Modulationen«, die in solchen Vorspielen »recht gut an ihrem Platz« seien. ${ }^{27}$ Er warnt gleichwohl, dass sie nur bei »gründliche[r] Harmonie-Kenntniss « zu bewältigen seien, welche er voraussetzt. Die vorgestellten Beispiele lassen sich als ein verkapptes Modulations- und Ausweichungskompendium verstehen - insbesondere jener avancierten chromatisch-enharmonischen Möglichkeiten, wie sie etwa in der Musik Schuberts und Beethovens der 2oer-Jahre zu finden sind.

In Exempel I3 demonstriert er die modulatorische Flexibilität des verminderten Septakkordes, indem eine neapolitanisch angereicherte Kadenz in a-Moll auf dis, der doppeldominantischen Bassstufe \#(4), als Fermate zum Stehen kommt und dann als doppeldominantische Bassstufe (6) nach fis-Moll umgedeutet wird (Notenbeispiel 3a). Die enharmonische Verwechslung des c ins his und damit die Verwandlung des klangidentischen Akkordes von der Sept- in die Quintsextposition stellt Czerny dabei allerdings nicht in Noten dar.

Weitere chromatisch-enharmonische Beispiele wie Exempel I4 (chromatische Verbindung eines Des-Dur-Quartsextakkords hin zu einem D-Dur-Sextakkord bei verminderter Terzfolge im Bass in Takt 2 f.), Exempel i6 (chromatische FauxbourdonRückungen) oder die längeren Exempla I8 und I9 mit ihren Varianten und mediantischen Tonbeziehungen wären hier ebenfalls anzuführen.

Exempel I5 (Notenbeispiel 3b) führt den neapolitanischen Sextakkord als Modulationsmittel im B-Dur-Kontext des Beginns ein. Der als chromatische Rückung in Takt 3 erreichte Ces-Dur-Sextakkord wird dabei mittels der Oktavregelprogressionen (5) 6- (4) ${ }^{2-}$ (3) ${ }^{6}$ temporär tonikalisiert (Czerny notiert enharmonisch H-Dur in Takt 5), um in Takt 5 mediantisch in eine angedeutete g-Moll-Kadenz zu leiten und dann schließlich in Es-Dur zu kadenzieren. ${ }^{28}$

Erst im letzten Beispiel (Notenbeispiel 3c) des Kapitels erläutert Czerny rudimentär das Prinzip der Umdeutungsmodulation und insbesondere der enharmonischen Verwechslung. Allerdings wird dies nicht in Worten ausgeführt, bis auf die kurze Bemerkung, dass sich »die enharmonischen Accorde zu mannigfaltigen Schlusswendungen« »vorzüglich eignen ${ }^{29}{ }^{29}$ Stattdessen findet sich ein Notenbeispiel mit zwei alternativen Fortgängen, die im Text markiert sind. Zum einen wird bei der Markierung (A.) ein verminderter Septakkord aus seinem C-Dur-/a-Moll-Kontext enharmonisch umgedeu-

Czerny: Systematische Anleitung zum Fantasieren, S. 9.

28 Der Dominantseptakkord D7in Takt 5 wird dabei zunächst unweigerlich als übermäßiger Quintsextakkord gehört, da die Spreizung der Außenstimmen das Modell Sexte $\rightarrow$ übermäßige Sexte $\rightarrow$ Oktave nahelegt. Czerny zeigt hier bereits die modulatorische Beweglichkeit des übermäßigen Quintsextakkordes, die er erst im letzten Beispiel 20 erläuternd ausführt. 

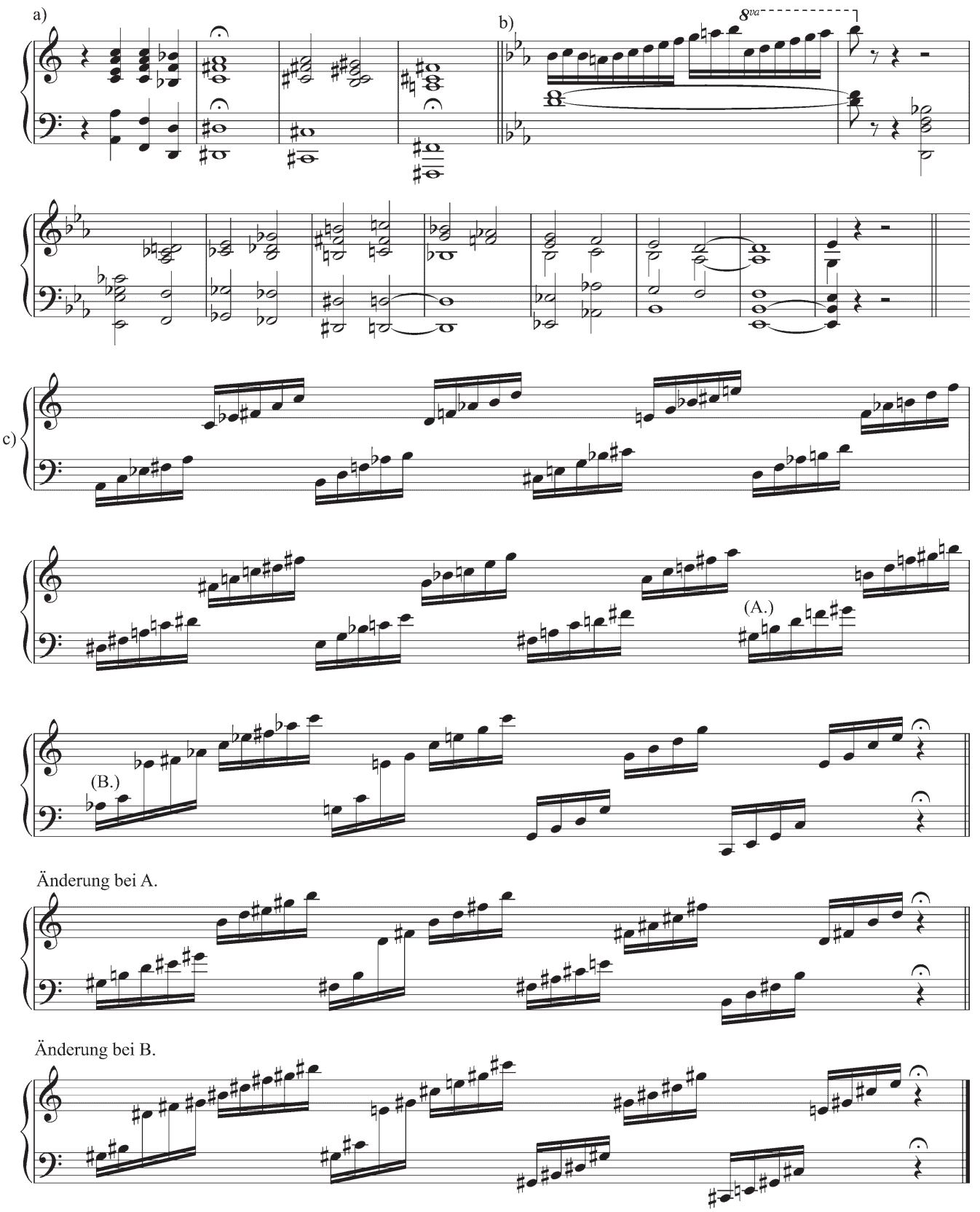

Noten BeISPIEL 3 Enharmonische und chromatische Relationen und Modulationen in Czernys op. 200

a) Exempel 13, S. 10; b) Exempel 15, S.10;

c) Exempel 20, S. $13 \mathrm{f}$. 
tet und mit neuem Leitton eis doppeldominantisch in eine h-Moll-Kadenz umgelenkt, die zweite »Änderung bei B « stellt die Klangidentität des übermäßigen Quintsextakkordes mit dem Dominantseptakkord vor. ${ }^{30}$

In fast allen Beispielen zeigt sich eine Vorliebe für den Einsatz des verminderten Septakkordes, teilweise auch in mehrfacher direkter Folge, was zu voll-chromatischen Klangfeldern führt(Notenbeispiel 3c). Die Bedeutung des Akkords für das Improvisieren ist naheliegend und lässt sich auch in improvisatorischen Genres der Barockzeit, etwa in Präludien und Toccaten, mehrfach nachweisen: Der Akkord ist jederzeit einsetzbar, flexibel und wandlungsfähig. Er ist gewissermaßen der Inbegriff tonaler Unbestimmtheit und Freiheit und kann im Moment der Klangerfindung sichere Zuflucht bieten, sollten der rote Faden sich verlieren oder die Ideen verebben. Für Czernys Zeit rückt, wie diese Beispiele deutlich zeigen, insbesondere jene modulatorische Freiheit ins Zentrum des Interesses.

Die Basis, das Orientierungsgefüge für diese harmonische Ausgestaltung, bilden neben den Kadenzverläufen und ihren Erweiterungen modellhafte Bassverläufe und Sequenzmuster, die in fast jedem Beispiel zur Anwendung kommen und den größten Teil der Exempel ausmachen. Hierdurch ist die Improvisation auf die kombinatorische Freiheit reduziert, im Moment aus einem Repertoire verschiedener Kadenz- und Sequenzmuster zu wählen. Dies legen auch Czernys abschließende Äußerungen für das Kapitel nahe. Er empfiehlt darin, die mittels der Beispiele angeeigneten Passagen mit Partien aus eigenen oder fremden Werken, die dem Spieler gleichsam als Vokabeln oder Phrasen zur Verfügung stehen, wie Züge im Würfelspiel aneinanderzusetzen: »Natürlicherweise muss man diese und ähnliche Beyspiele in alle Tonarten übersetzen, die Passagen mit andern schicklichen abzuwechseln «.3 ${ }^{\mathrm{I}}$ Der improvisatorische Gestus wird demnach als Arrangieren im Moment über freie Kombinationsmöglichkeiten aus erlernten Formeln und Mustern in Harmonik und Figuration erreicht. Nur dürfe man sich dabei nicht zu starr an bestimmte Muster, an ein immer gleiches »Preludier-Formular« halten, damit man nicht »fast jedesmal das Nämliche zu hören bekommt. «32

Notenbeispiel 4 zeigt vier solche Bassverläufe in Generalbassreduktion und mit kurzen, analytischen Kommentaren (ohne Czernys Akkordfiguration). Czerny demonstriert sowohl ein breitgefächertes Arsenal an Mustern als auch eine flexible Anwendung derselben in diesem Kombinationsdenken. Sie sind dabei fast sämtlich chromatisch

Der übermäßige Dreiklang spielt bei Czerny in diesem Kontext noch keine Rolle, er wird erstmals von Carl Friedrich Weitzmann theoretisch beschrieben; vgl. ders.: Der übermässige Dreiklang, Berlin 1853 .

31 Czerny: Systematische Anleitung zum Fantasieren, S. 9.

32 Ebd., S. I4. 
a)

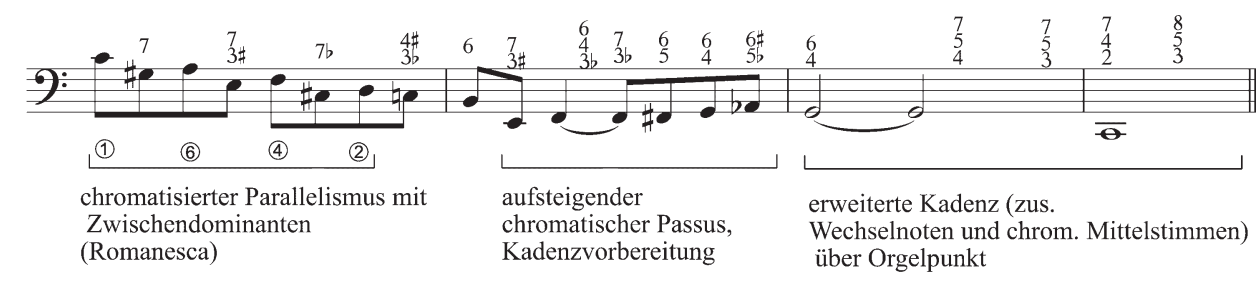

b)

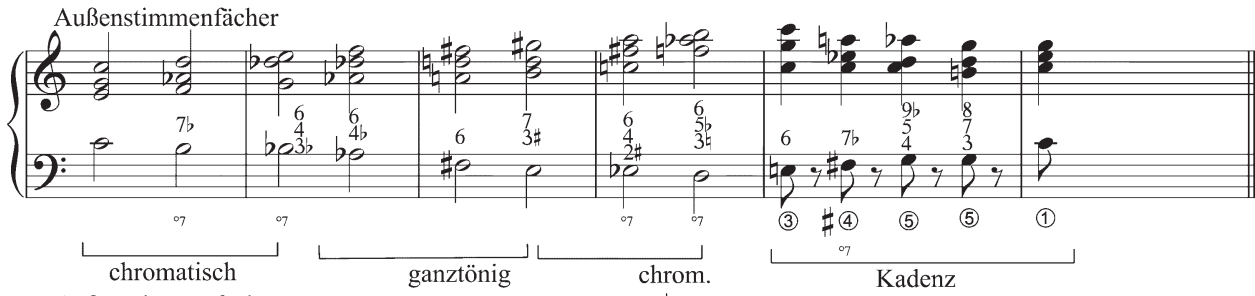

c)

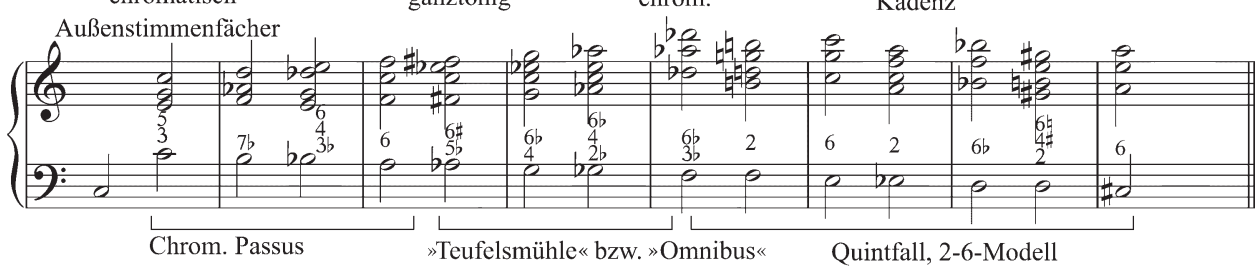

d)

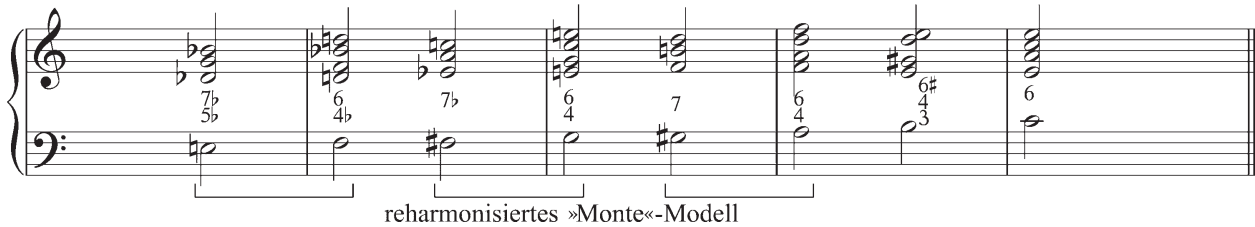

NOTEN BEISPIEL 4 Bassverläufe in Czernys op. 200: Musterbildungen und Topoi

a) Exempel 5, S. 7; b) Exempel 14, S. 10; c) Exempel 18 (Takt 1-9), S. 12;

d) Exempel 19 (Takt 8-12), S. 12

erweitert, meist mit verminderten Septakkorden versehen, und vermitteln eine musikalische Sprache auf der Höhe der Zeit: Die Parallelismus-Sequenz (4a, Takt rff.), die Riepel'schen »Fonte«- (4c, Takt 5 ff.) und »Monte«-Modelle, terzweise fallende Quintfallsequenzen, »Teufelsmühlen «-Modelle und Außenstimmenfächer werden in komplexen modulierenden Zusammenhängen präsentiert.

Neben den barocken Grundmodellen, wie sie die Partimento-Schulen des I8. und frühen I9. Jahrhunderts noch lehren, finden sich zahlreiche zeitgenössische Neuerungen. So ist im chromatischen Bass-Anstieg in Notenbeispiel $4 \mathrm{~d}$ das im Hintergrund wirksame »Monte«-Modell noch klar zu erkennen. Der übliche Quint- oder Quintsextakkord zu Beginn eines jeden Sequenzgliedes ist allerdings durch den verminderten Septakkord ersetzt und das Ziel ist nicht mehr ein grundständiger Terzquintakkord, sondern ein Quartsextakkord (vergleiche Notenbeispiel 4d). Auch wenn der Bassgang erhalten bleibt, die Akkordfolge verändert sich dadurch beträchtlich. Der ursprüngliche 
Quintfall ist nicht mehr vorhanden, aus der Folge $\mathrm{C}^{7} / \mathrm{E} \rightarrow \mathrm{F}$ und $\mathrm{D}^{7} /$ Fis $\rightarrow \mathrm{G}$ wird $\mathrm{e}^{\circ 7} \rightarrow \mathrm{B} / \mathrm{F}$ und fis ${ }^{\circ 7} \rightarrow \mathrm{C} / \mathrm{G} .33$

Einige Beispiele gehen noch einen Schritt weiter und lösen sich von den standardisierten Abfolgen (insbesondere die Exempla I8 und I9 auf Seite I2). In ihnen verläuft nur noch der Bass musterhaft, zumeist skalar oder chromatisch auf- oder absteigend, die harmonische Ausgestaltung darüber ist frei und folgt nicht oder nur teilweise modellhaften Klangmustern.

Hierin lässt sich ein Grundcharakteristikum des fortgeschrittenen Improvisierens erkennen: Um die gewünschte Freiheit und Flexibilität der Erfindung im Moment zu erreichen und nicht bei bloßer Kombinatorik von Vorgelerntem zu verbleiben, muss die Konzentration auf Ausgestaltung der Harmonik und Figuration liegen. Und um dieser Freiheit gerecht werden zu können, ist der übergeordnete Verlauf durch ein gewähltes Muster vorgegeben: Ein Bereich, beispielsweise eine chromatische Bassstimme, ist prädisponiert und als Orientierungslinie gegeben, um sich in anderen Bereichen frei bewegen zu können. ${ }^{34}$ Dabei kann das musikalische »Geländer« bisweilen auch in andere Stimmen verlegt werden, etwa in die Oberstimme, in beide Außenstimmen als Fächermodell (Notenbeispiel 4b, Beginn) oder in eine Mittelstimme (Exempel I7 im Tenor über dem Orgelpunkt im Bass).

Czernys Vorgehen ist sowohl bei den Kadenz- als auch bei den komplexeren Modulationsverläufen zweischrittig, hinterlässt jedoch hinsichtlich der genauen Methodik (wie sie im praktischen Einzelunterricht erfolgen würde) mehrere Leerstellen: Zunächst werden im einfachen Akkordsatz Klangfolgen gelernt und diese in alle Tonarten übertragen (Czernys Beispiele beginnen durchweg in C-Dur und a-Moll). Dann folgen unterschiedliche Möglichkeiten der figurativen Ausgestaltung, indem unterschiedliche Akkordbrechungen und Lauffiguren vorgestellt werden (Notenbeispiel 5). Dabei ist es ihm wichtig zu betonen, dass sich »aus jedem Accord [...] die mannigfaltigsten Passagen entwickeln lassen «.35 Zunächst sind diese meist auf ein gleichbleibendes Bewegungsmodell ausgerichtet, die »Preludien längerer und mehr ausgeführter Art« (Kapitel 2, S. I5ff.) präsentieren mannigfaltige Abwechslungen aus chromatischen Läufen, Wechselnoten- und Trillerfiguren, sowie gebrochene Akkorde (häufig mit chromatischen Einfügungen).

Czerny stellt den verminderten Akkord $\mathrm{e}^{\circ} 7$ enharmonisch als cis ${ }^{\circ 7}$ dar; vgl. Exempel ig (Takt 8, Ziffer 2), S. I2.

34 Dies lässt sich in auffälliger Häufung auch noch in auskomponierten Fantasien, gewissermaßen als sedimentierte Improvisation beobachten. Bekanntes Beispiel ist der chromatisch absteigende Beginn in Mozarts c-Moll-Fantasie KV 475.

35 Czerny: Spstematische Anleitung zum Fantasieren, S. 6. 

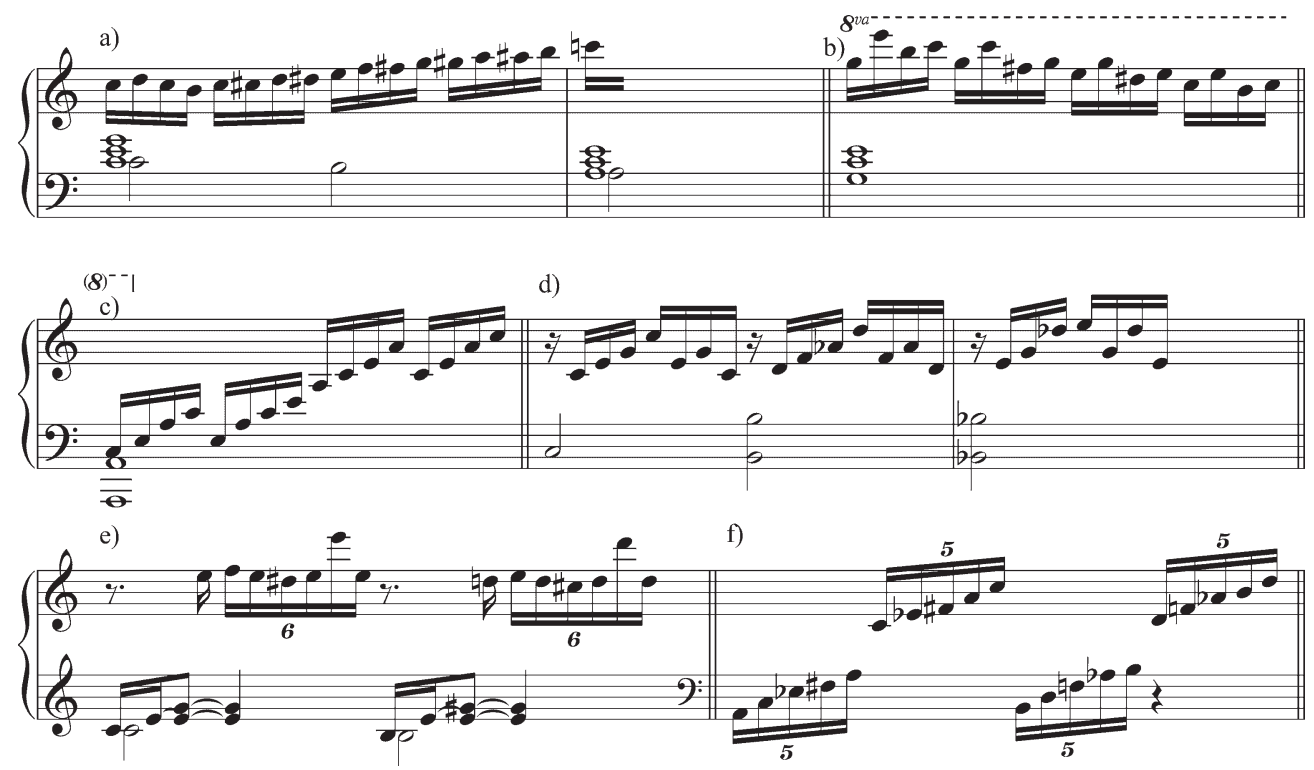

NOtEN BE IS P IEL 5 Figurationsmodelle: Lauffiguren und Arpeggien in Czernys op. 200 (Auswahl)

a) Exempel 3, S.6; b) Exempel 3 (Takt 5), S.6; c) Exempel 4, S. 7; d) Exempel 14, S. 10;

e) Exempel 16, S. 10; f) Exempel 20, S.13

Czernys Beispiele sind jedoch immer komplett ausnotiert und enthalten keine Aufgaben oder Übungen zum Harmonisieren oder Figurieren, etwa bezifferte und unbezifferte Bassverläufe, wie sie aus Improvisationslehren (zum Beispiel noch bei Joseph Drechs$\left.{ } \mathrm{er}^{36}\right)$ und der italienischen Partimento-Tradition selbstverständlich sind. So ließen sich, ginge man nur von diesem Lehrwerk aus, die Akkordverbindungen einfach exakt nachspielen und transponieren; das eigene harmonische Ausführen nach Stimmführungsregeln in unterschiedlicher Stimmenanzahl, Satztechnik, Oktavregistern und Lagen wird nicht gelehrt, sondern es wird vorausgesetzt. Insofern geht Czerny von einem idealtypischen Schüler aus, der neben dem Generalbass bereits sämtliche Figurationsmuster und Skalenbildungen erlernt hat und diese nun selbständig über die gegebenen (idealer noch: eigenen) Bässe anwendet. So geht er einerseits über die meisten Partimentolehren des I8. und I9. Jahrhunderts hinaus, indem er harmonisch komplexere Verläufe verlangt, bleibt andererseits jedoch methodisch hinter dem klaren Aufbau und der bestechend einfachen wie erfolgreichen Methode zurück.

Es mag sein, dass der Autor diesen Mangel an methodischer Führung selbst empfunden hat, denn in der Klavierschule op. 500 reagiert Czerny mit einer genaueren Ausführung zum methodischen Vorgehen. Im Kapitel »Über das Präludieren« findet sich

36 Joseph Drechsler: Theoretisch-praktischer Leitfaden, ohne Kenntniß des Contrapunctes phantasieren und präludieren zu können. Als Anleitung zu den öffentlichen Vorlesungen in der Harmonielehre und dem Orgelspiel, Wien [1834]. 
dort ein basaler Ansatz, den er wohl erst nach dem Verfassen der Improvisationsschule entwickelt hat und der zeigt, wie die Übungen zur Erlangung technischer Sicherheit und zum Improvisieren nicht in zwei getrennten Schritten, sondern Hand in Hand gehen können. Rudimentäre Präludien sollen hier aus den vorgeschlagenen »Scalenübungen« in Verbindung mit simplen V-I-Kadenzen gebildet werden.

»04. Selbst der Anfänger kann und muss bereits in den ersten Monathen dazu angehalten werden, vor jedem Tonstücke ein kleines Vorspiel auszuführen, und auch hier sind die Scalenübungen das Erste und vorzüglichste Hilfsmittel.

05. Diese Scalenübungen werden hiezu [sic] folgendermassen verwendet:

a.) Man spielt in der Tonart des nachfolgenden Tonstückes eine, oder mehrere der allda vorkommenden Passagen mit der rechten Hand allein, während die Linke den Grundton hält.

b.) Oder man spielt eine oder mehrere dieser Passagen zuerst auf dieselbe Weise mit der rechten Hand allein, und hierauf mit beiden Händen. Die Passagen können in beliebiger Ordnung nacheinander folgen.

c.) Oder man spielt alle, in der betreffenden Tonart vorkommenden Passagen vollständig genau so, wie dieselben in der grossen Scalenübung gelernt worden sind.

[...] Man sieht, dass sich jeder Schüler, mit einigem Nachdenken, leicht eine Menge solcher einfachen Vorspiele schon aus den Scalenübungen selber bilden kann«37

Interessant ist dabei, dass er - anders als in op. 200 - bereits im relativ frühen Stadium der pianistischen Ausbildung dieses rudimentäre Improvisieren empfiehlt, das vollständig aus einem Kombinieren erlernter Muster besteht.

Dass er hier grundständiger als in op. 200 vorgeht, zeigt sich auch daran, dass nun eine Tafel mit den »zwei Schlussakkorden« in allen 24 Tonarten folgt, die den Abschluss dieser einfachen Präludien bilden. Die Mühe, Beispiele zu transponieren, macht sich Czerny in op. 200 nicht, sondern fügt die obligatorische Lehrbuchempfehlung hinzu, man solle die Beispiele in allen Tonarten üben; die sichere Kenntnis des Generalbassspiels setzt er, wie erwähnt, ohnehin voraus.

»Selbstständige« Fantasien - Fantasia quasi una Sonata Dass nun das reine Akkord- und Skalenspiel nicht ausreicht, sondern Improvisationen »einen regelmässigen Zusammenhang« besitzen, und »Passagen« sich mit »Gesangstellen« abwechseln müssen, wird bereits im zweiten und dritten Kapitel klar, wo es um längere Präludien und Solokadenzen geht. ${ }^{3}$ Solokadenzen in Klavierkonzerten begreift Czerny dabei "gewissermassen als selbstständige Fantasien «, die er aus systematischen Gründen vorschaltet.39 Erst im 4. Kapitel »Vom Fantasieren über ein einzelnes Thema« liest man Details über die eigentliche >Kunst ` des Improvisierens. In späteren Lehrwerken werden die Bereiche 
ganz getrennt: In der Klavierschule op.500 behandelt das I8. Kapitel das »Präludieren«, das damit auch begrifflich vom »Fantasieren (oder Improvisieren)« im darauffolgenden Kapitel abgekoppelt ist, gewissermaßen als dessen Vorstufe.

Entscheidend sind für Czerny dabei zwei Bereiche: die Gestaltung des Themas, seine flexible Umgestaltung und die Bedeutung motivischer Verarbeitung (behandelt in den [פ $\mathrm{I}-7)$ und die Herausbildung eben jenes »regelmässigen Zusammenhanges«, der Form, die der Improvisation eine kompositorische Qualität verleiht (פg 8-I5). Da dieses Kapitel den zentralen theoretischen und methodischen Kern bildet, der dann auf die übrigen Kapitel übertragen wird (Kapitel 5 »Freyeres Fantasieren über mehrere Themas«, Kapitel 6 »Potpourri«, Kapitel 7 »Variationen «), sei auf dieses, insbesondere die beiden darin enthaltenen exemplarischen Fantasien, näher eingegangen. Diese stellen sicherlich einen Sonderfall hinsichtlich der Fokussierung auf ein einziges thematisches Ausgangsmaterial dar, abgesehen von dieser Besonderheit weichen sie aber von den Beispielen über mehrere Themen in den Kapiteln fünf und sechs nicht wesentlich ab.

Inwieweit diese »Ite Gattung « des »selbstständigen « Fantasierens in Wien verbreitet war, lässt sich schwer beantworten, eine nennenswerte Geltung und Bekanntheit als eigenständige Improvisationstradition lässt sich durchaus bezweifeln. Sicherlich war sie zum Erscheinungszeitpunkt von Czernys Schrift kaum mehr im Gebrauch. Er selbst empfiehlt beim öffentlichen Fantasieren die Gattung des Potpourris, worunter sich insbesondere die Opernfantasie zählen lässt, und rät beim öffentlichen Fantasieren von Improvisationen über eigene Themen (insbesondere über ein einziges) ab. Nur die größten Improvisatoren seien laut Czerny dieser Herausforderung gerecht geworden, ohne das Publikum zu ermüden oder zu langweilen..$^{40}$ So kann er hier auch im Gegensatz zu allen anderen Kapiteln keine Beispiele komponierter Fantasien nennen und verweist hinsichtlich der formalen Gestaltung auf Sonaten klassischer Autoren: »das erste S[t] ück einer guten Sonate (von Clementi, Beethoven, Hummel \&)«.4 ${ }^{\mathrm{I}}$

Struktur und Tonalität: Improvisation und die »double function form« Sonatenform, motivisch-thematische Arbeit und Improvisation sind für Czerny keine Widersprüche, im Gegenteil: Er geht selbstverständlich von der Sonate als gedanklicher Basis aus, wie

40 Ebd., S. 43. Aus methodischer Sicht stellt sich zu Recht die Frage, warum er Themen- und Formbildung nicht an der einfacheren und populäreren Form der Variationen ausführt, siehe oben.

41 Ebd., S. 42. In einer Anmerkung auf S. 43 nennt er zudem zwei Musterbeispiele, die bezeichnenderweise keine Klavierkompositionen sind: Beethovens Chorfantasie op. 80 und das Finale der 9. Sinfonie op. I25. Er führt in diesem Zusammenhang überraschenderweise auch Bachs Kunst der Fuge Bwv ro80 an. Vgl. zu Czernys »Fantasieren über ein einzelnes Thema« auch den Beitrag von Martin Skamletz über Joseph Lipavsky in diesem Band, insbesondere die Betrachtung zu dessen Rondo op.30 (S. I37-I63). 
beide Beispielfantasien des Kapitels zeigen. Dies deckt sich durchaus mit den komponierten Klavierfantasien der I8Ioer- und 2oer-Jahre, etwa der äußerst bekannten Fantasie Es-Dur Hummels (die Czerny als kompositorisches Vorbild in Kapitel fünf empfiehlt) und weicht von älteren, rhapsodischen Typen des I8. Jahrhunderts in der Tradition der freien Fantasie C. P. E. Bachs ab. Mozarts Beiträge zur Gattung, etwa das Capriccio C-Dur KV 284a, partiell ohne Taktordnung notiert, sind dafür ein Beispiel, ebenso die beiden bekanntesten Mozart-Fantasien d-Moll Kv397 und c-Moll Kv 475. Letztere hebt sich dabei als großes, quasi-improvisatorisches Präludium bewusst von der nachfolgenden Sonate KV 457 ab und vermeidet eine Nähe zur Sonatenform. ${ }^{42}$

Im frühen I9. Jahrhundert ist die gegenseitige Annäherung der Gattungen Fantasie und Sonate jedoch unaufhaltsam, 43 bis zur schlussendlichen Austauschbarkeit der Begriffe in der Romantik, wie sie in Robert Schumanns bekannter Aussage deutlich wird: »Also schreibe man Sonaten, oder Phantasien (was liegt am Namen), nur vergesse man dabei die Musik nicht« 44 Dass diese beiderseitige Durchdringung dabei nicht nur die komponierte Musik, sondern auch die Improvisation betrifft, findet allerdings kaum Erwähnung. Auf gleiche Weise werden Formkonzepte der komponierten Musik zur Strukturierung der Improvisation genutzt und damit gleichzeitig der hohe Anspruch hinsichtlich der strukturellen Gestaltung und Übersicht während des Spiels unterstrichen. Damit konnten große Formen sinnvoll und abwechslungsreich nach dem Tonalitätsverständis der Zeit gestaltet werden, um das Publikum mit den vertrauten, jedoch nie sich werkhaft verfestigenden Ordnungsgefügen im ad-hoc-Spiel zu verblüffen. Das hat erhebliche Konsequenzen für das künstlerische Verständnis von »Freiheit« und »Konstruktion « - sie sind nicht einfach mit Improvisation und Komposition gleichzusetzen. So wirken einige Passagen in Mozarts Fantasien im Vergleich zu Czernys »Fantasieprotokollen « viel freier in ihrer quasi-improvisatorischen Fluidität, sie basieren jedoch auf einer wohlkonstruierten Anlage, die Czernys Beispiele nicht aufweisen: Die Wiederholungen ganzer Teile und Themenpassagen sind einer kompositorischen Planung geschuldet, die improvisatorisch nur sehr schwer möglich beziehungsweise nur bei außergewöhnlicher Gedächtnisleistung zu erreichen wäre. Czerny bedenkt auch diesen Umstand: »Repetitionen sind im Fantasieren nicht wohl möglich, denn selten bleibt das

42 Die vielfachen Versuche, sie dennoch als Sonatenform zu deuten, überzeugen allesamt nicht. Sie zwängen das Stück unter Weglassung entscheidender Kriterien in die Form und verstricken sich in unauflösbare Widersprüche; vgl. etwa Edler: Gattungen der Musik für Tasteninstrumente, Teil 2, S. 79 .

43 Ansätze dieses Trends sind schon bei C.P. E. Bach zu finden; vgl. ebd., S. 56-7I.

44 Robert Schumann: Sonaten für das Clavier, in: Neue Zeitschrift für Musik ıo (ı839), Nr. 34, S. ı34f., hier S. I34. 
eben Gespielte so lange im Gedächtnisse.«45 In diesem Sinne sind Czernys Beispiele, obgleich »logisch « sonatenhaft konzipiert, näher an einer realistischen Improvisation als scheinbar freiere, auf improvisatorischem Gestus beruhende, aber doch komplexer gestaltete komponierte Fantasien.

Komposition und Improvisation durchdringen sich also gegenseitig in dem Sinne, dass komponierte Musik improvisationsartige Elemente zur Erneuerung von Strukturen und Erweiterungen dur-moll-tonaler Beziehungen einsetzt (etwa in Beethovens Sonaten op. 27 »quasi una fantasia«). Umgekehrt findet sich aber auch das Bemühen, in improvisierter Musik quasi-kompositorische Zusammenhänge zu suchen und das Verhältnis von offenen und ungebändigten Passagen (»a capriccio«) mit festgefügten, geschlossenen Formen im Wechsel neu auszutarieren.

In op. 200 gibt Czerny einige konkrete Hinweise zur Form einer solchen großen Improvisation: Als Vorübung solle man Einzelsätze vollständig »durchführen«. Neben Allegrosätzen in Sonatenform verlangt Czerny dabei die Beherrschung von langsamen Sätzen, Scherzi, Rondi und Variationen - sämtlichen Satzarten des Sonatenzyklus. Den Sonatensatz erklärt er dabei ausführlicher: dem »Hauptthema« müsse ein »passende[r] Mittelgesang«, also der Seitensatz, folgen, auch die üblichen Tonartenverhältnisse werden genannt. Der »erste Theil«, also die Exposition, werde dabei wie in komponierten Werken abgeschlossen, im »2ten Theil« (Czerny sieht die Sonate noch als zweiteilige Form) könne man »sich der freyesten Fantasie und Ausführung, und allen Arten von Modulationen, Imitationen \&, völlig überlassen«. Die Durchführung bietet demnach wieder mehr Platz für »Passagen und ander[e] Figuren « ${ }^{46}$ jedoch müsse man sich der »Gesangstellen« (Czerny schließt den »Mittelgesang« des Seitensatzes ein) wieder erinnern, um »endlich in der Haupttonart « zu schließen. ${ }^{47}$ In der Verbindung mit anderen Satztypen scheint das jedoch nicht mehr zu gelten, wie die Beispiele zeigen: Czerny nähert sich dort zwar wieder der Ausgangstonart an, allerdings um die Kadenz zu vermeiden und in die neue Tonart des zweiten Satztyps zu wechseln (vgl. Exempel 42, Takt 65-76). Die Sonatenform bricht in diesem Falle also vor dem Eintritt der Reprise ab und leitet in die neue Tonart des nächsten Satzteiles über. Zur Gesamtform gibt er ebenso nur einige kurze Hinweise. Hier solle man sich bemühen,

»mehrere Gattungen in einer und derselben Fantasie zu verbinden. Man fange z. B. mit Allegro an, durchführe eine Zeit lang, gehe dann in ein Adagio oder Andantino über, durchflechte es mit einem

Czerny: Spstematische Anleitung zum Fantasieren, S. 55 .

46 Edler sieht in solchen fantasie-typischen freien und modulatorischen Passagen einen entscheidenden Einfluss für die Entstehung der Durchführung als ausgeprägtem, eigenständigem Formteil in Sonaten; vgl. ders.: Gattungen der Musik für Tasteninstrumente, Teil 2, S. 70. 
fugierten Satz und mit den, in den ersten Kapiteln besprochenen modulierenden Sätzen [die beiden Kapitel zum Präludieren, Anm. d. Verf.], und ende mit einem lebhaften Rondo.« $4^{8}$

Czernys Idee einer großen, durchgehenden Anlage in verbundenen Einzelsätzen vereint also einen sonatenhaften Kopfsatz mit einem langsamen Satz und einem abschließenden Rondo. Diese einer dreisätzigen Sonate entsprechende Großform enthält jedoch vor dem Rondo im Schlussteil des Adagio »modulierende Sätze«, wie beispielsweise einen »fugierten Satz«, sodass die Form gleichzeitig als einsätzige Sonatensatzform mit Durchführungsteil und Rondo für die tonale und thematische Reprise begriffen werden kann. Czernys Beschreibung lässt sich als »double function form« interpretieren, welche die musikwissenschaftliche Formenlehre üblicherweise erst seit Franz Liszts symphonischen Werken und den großen einsätzigen Klavierwerken datiert. ${ }^{49}$ Dieser Formtypus wird meist der kompositorischen Entwicklung der romantischen Klavier- und Orchestermusik zugeordnet, Detlef Altenburg beschreibt im Falle Liszts das Konzept der symphonischen Dichtung als Verschmelzung der einsätzigen Ouvertüre und der mehrsätzigen Programmsinfonie. ${ }^{\circ}$

Statt die »double function form« von den Kompositionen Liszts herzuleiten, kann man mit Czerny eine andere Wurzel vermuten, nämlich die Klavierimprovisation zusammenhängender Tonstücke mit mehreren Satzfolgen, die im Zuge der Annäherung von Sonatenformen und Fantasien auf Klavier und Orchesterwerke übertragen wurde. Dafür spricht auch die Tatsache, dass Liszt - überdies Schüler Czernys - vor den großformalen Kompositionen in Weimar in den I83oer- und frühen I84oer-Jahren zahlreiche Klavierfantasien (meist Opernfantasien ${ }^{5}$ ) schrieb und allgemein für seine stupende

48 Czerny: Systematische Anleitung zum Fantasieren, S. 43.

49 Vgl. zu Liszt und der »double function form«William S. Newman: The Sonata since Beethoven, Chapel Hill I969; Kenneth Hamilton: Liszt. Sonata in B Minor, Cambridge I996 (Cambridge Music Handbooks), S. 28; Steven Vande Moortele: Two-Dimensional Sonata Form. Form and Cycle in Single Movement Instrumental Works by Liszt, Strauss, Schoenberg, and Zemlinskp, Leuven 2009, S. 20 ff.; Jay Rosenblatt: Piano and Orchestra Works, in: The Liszt Companion, hg. von Ben Arnold, Westport 2002, S. 28I-307, insb. S.302. Rosenblatt deutet bereits Werke der 3oer-Jahre als frühe Kompositionen in »double function form«, etwa De profundis von I834/35.

50 Detlef Altenburg: Franz Liszt und das Erbe der Klassik, in: Die Geschichte der Musik, Bd.3: Die Musik der Moderne, hg. von Matthias Brzoska und Michael Heinemann, Laaber 200I, S. I-I9, S. I9.

51 Die Opernparaphrasen folgen formal eher dem von Czerny vorgestellten Potpourri-Typus mit einem Fokus auf Variationssätze. Sie gehen aber in ihrer formalen Anlage und ihrer Beziehung zur Opernvorlage weit über Czernys Ansatz hinsichtlich einer Verdichtung und Relektüre des dramatischen Verlaufs hinaus; vgl. Charles Rosen: The Romantic Generation, Cambridge I995, S. 528. Vgl. zur Bedeutung der frühen Fantasiekompositionen für die späteren Orchesterwerke Michael Saffle: Liszt and the Traditions of the Keyboard Fantasy, in: Liszt the Progressive, hg. von Hans Kagebeck und Johan Lagerfelt, Lewiston 200I, S. I5I-I86. 
Improvisationskunst bekannt war - in einer Zeit, in der diese Kompetenz allmählich nicht mehr obligatorisch war. Liszt war zudem nachhaltig beeindruckt von Franz Schuberts Wanderer-Fantasie, die ihn zur kompositorischen Revision inspirierte und als frühes Beispiel dieses einsätzigen Formtypus' der Fantasie genannt werden kann.

Betrachtet man nun die beiden Beispielfantasien in Czernys op. 200, so führen sie dieses Modell zwar aus, jedoch im Gegensatz zu Czernys einführenden Worten in der Viersätzigkeit. Tabelle 2 zeigt eine formale Übersicht der beiden Beispielfantasien Exempel 42 und 43 in Kapitel 4 (S. 44-53 und 53-62). Die gefestigten Satzteile mit stabiler Tonalität und jeweils zu Beginn klarer Satzstruktur ${ }^{2}$ werden dabei vermittelt und eingefasst von passageren und tonal beweglichen Teilen. Beide Fantasien werden gerahmt, indem die Präludienstruktur des Anfangs in verkürzter Form wiederkehrt und in die letzten Takte der finalen Rondoteile eingefügt beziehungsweise als Codetta angehängt wird. Czerny bleibt seiner Ankündigung treu und belässt, was Wiederholungsteile betrifft, eine realistische Situation: Nur klare Viertakt-Strukturen erfahren Wiederholungen, zudem zumeist direkt hintereinander. Über große Zeitabstände hinweg bleibt nur die Wiederholung des Motivs als Einheit stiftendes Moment präsent, die Formung durch annähernd exakte oder wörtliche Wiederholung findet nicht statt. Czerny wählt konventionelle Zusammenhangsbildung durch Rückkehr in die jeweilige Ausgangstonart, der jeweilige Schlusssatz bietet zusätzlich zu dieser tonalen Reprise wieder die größte Annäherung an das Grundmotiv, wie es im ersten Allegrosatz auftrat. Im Falle der ersten Fantasie geschieht dies durch die Übernahme des punktierten Grundmotivs und dessen Überführung in eine aufsteigende Romanesca-Sequenz, im zweiten Beispiel ist das prägnante Quartmotiv mit folgender Punktierung nun nicht mehr in der Oberstimme, sondern im Bass. Dadurch besitzen die abschließenden Rondoteile53 Reprisenqualität im Sinne der »double function form«. Beide Kopfsätze modulieren gemäß der Sonatenform in die quinthöhere beziehungsweise im c-Moll-Kontext des ersten Beispiels in die parallele Durtonart und bilden dort einen »Mittelgesang« auf Basis des Grundmotives aus (Exempel 42, Takt 53 ff.; Exempel 43, Takt 2I ff.). Beide brechen dann allerdings den Sonatenverlauf ab und fügen statt der bekräftigenden Schlussgruppe des Expositionsteils eine modulatorische Passage als Übergang zum nächsten Satztypus ein (in Exempel 43 bezeichnet als à capriccio, Takt 3I).

Einzige Ausnahme ist der Agitato-Teil anstelle des Scherzos in Exempel 42, der von Beginn an transitorische Gestalt hat.

53 Das Sonatenrondo ab S. 50 ist nur angedeutet, besteht nur aus einer dreiteiligen ABA-Form mit Coda. Der в-Teil ist gleich in modulatorischer Rückführung gesetzt. Czerny selbst empfiehlt den Lesern die eigene Ausarbeitung und Verlängerung bei eigenen Übeversuchen (S. 5 I f.). 


\begin{tabular}{|c|c|c|c|c|c|}
\hline \multirow[t]{5}{*}{ Ex. 42 (Kap. 4) } & Lento, quasi Andante & Allegro con brio & Agitato & Adagio espressivo & Allegro Vivace \\
\hline & T. I-I2 & T. $13-76$ & T. $77-\mathrm{II} 3$ & T. II4-I52 & T. $153-263$ \\
\hline & $4 / 4$ & $3 / 4$ & $3 / 4$ & $2 / 4$ & $2 / 4$ \\
\hline & $\mathrm{c}$ & $\mathrm{c} \rightarrow \mathrm{Es} \rightarrow(\mathrm{c}) \rightarrow$ fis & fis $\rightarrow$ as(As) & As $\rightarrow \mathrm{a}$ & $\mathrm{c}$ \\
\hline & Präludium & Sonatenexposition & Scherzoartig & & Rondo \\
\hline \multirow[t]{5}{*}{ Ex. 43 (Kap. 5) } & Vivace & Allegro moderato & Presto & Adagio con moto & $\begin{array}{l}\text { Allegretto grazio- } \\
\text { so ed animato }\end{array}$ \\
\hline & Taktfrei & T. 2-33 & T. 34-I95 & T. I96-23I & T. $232-33 \mathrm{I}$ \\
\hline & & $4 / 4$ & $3 / 4$ & $4 / 4$ & $6 / 8$ \\
\hline & Es & $E s \rightarrow B \rightarrow d$ & $\mathrm{~d} \rightarrow \mathrm{D} \rightarrow \mathrm{E}$ & $\mathrm{E} \rightarrow \mathrm{Es}$ & Es \\
\hline & Präludium & Sonatenexposition & Scherzo & & \\
\hline
\end{tabular}

TABELLE 2 Formübersicht der beiden Beispielfantasien in Czernys op. 200, S. 44-62

In einem Punkt weicht Czerny jedoch von seinen schriftlichen Beschreibungen und der darin liegenden Lesart als Doppelfunktion der Form ab. Statt nach dem ersten Satz in ein Adagio überzugehen, schiebt er jeweils einen schnellen Satz im Dreiermetrum dazwischen. Im zweiten Falle ist das ein klarer Scherzo-Typus im Sinne Beethovens. Im ersten Falle ist dieser kein stabiler, eigenständiger Satz, sondern besitzt vielmehr die Funktion einer modulierenden Überleitung mit Durchführungsqualitäten. Es gibt zwar jeweils eine ebensolche Passage vor dem letzten Rondosatz, die damit dessen Bestimmung als Reprise verstärkt. Die Lesart des langsamen Satzes in der Funktion eines Seitensatzes in der Großform wird dadurch aber unterwandert.

In der tonalen Organisation der Einzelsätze zeigen die beiden Beispiele erwartungsgemäß die Vielfalt der Möglichkeiten, wie sie nicht nur komponierte Fantasien, sondern auch Sonaten der Zeit aufweisen. Das erste Beispiel ist vor allem durch Terzbeziehungen strukturiert ( $c \rightarrow$ Es $\rightarrow$ ges/fis; As $\rightarrow \mathrm{a} \rightarrow \mathrm{c}$ ), das zweite durch chromatische Umstellung des Zentraltones (Es $\rightarrow \mathrm{d} \rightarrow \mathrm{E}(\mathrm{Fes}) \rightarrow \mathrm{Es}$ ). Allerdings kehrt Czerny in allen Beispielen in die Ausgangstonart zurück und vermeidet tonale Entwicklungen, wie sie etwa Hummel in seiner Fantasie op. I8 vorführt, die in Es-Dur beginnt und in g-Moll/G-Dur endet. Nur im abschließenden Capriccio wählt Czerny einen ähnlichen Weg (Beginn in h-Moll, Schluss in C-Dur, S. I05-IIO).

Ein kurzer Blick auf das Beispiel des fünften Kapitels, eine Fantasie über mehrere Themen, zeigt, wie Czerny auch gleich im ersten Paragraphen deutlich macht, keine prinzipielle Verschiedenheit, aber eine formale Variante: Die unterschiedlichen Themen werden von einer thematischen Klammer, der punktierten Einleitungsmotivik, zusammengehalten. Auch dieses formale Verfahren ist bereits längst aus der Fantasie in komponierte Musik übergegangen, bekanntestes Beispiel hierfür ist Beethovens Sonate c-Moll 
op. 13 (1798/99). Czerny verlagert in diesem Beispiel den Ort des Sonatensatzes. Kürzere, gedrängte Formteile wechseln einander ab, der langsame Satz wird zum Variationssatz und das Finale in der Ausgangstonart d-Moll ist ein abschließender Sonatensatz mit verkürzter Reprise. Vorbild dafür könnten Beethovens Sonaten op. 27 »quasi una fantasia« sein, die beide erst in den Finalsätzen eine Sonatenform (beziehungsweise ein Sonatenrondo) aufweisen. Dafür spräche auch der formale Rekurs des langsamen Satzes, den Czerny als Anmerkung im Finalsatz vorschlägt. ${ }^{44}$ Genau wie in op. 27, Nr. I erscheint dieser in die Haupttonart transponiert und bei Czerny im Mollkontext zur Variante verwandelt.

Thematische Arbeit und Phrasengestaltung Zu Beginn des grundlegenden vierten Kapitels behandelt Czerny die Punkte Themengestaltung und -variabilität. Anhand einer viertaktigen melodischen Vorlage präsentiert er unterschiedliche thematische Ausgestaltungen, sie reichen von den üblichen Satzteilen einer Sonate bis hin zu Tänzen und Fugen. Dieselbe Variabilität präsentiert er im Folgenden an weiteren Ausgangsmaterialien, wobei die motivisch-thematische Vorlage unbestimmter wird. Ist das erste Beispiel (Exempel 38) selbst ein gestalteter Themenanfang, der die symmetrische Vordersatzstruktur bereits in sich trägt, ist das zweite Beispiel(Exempel 39) eine nicht-rhythmisierte Folge dreier Töne und Exempel 40 eine kurze »Figur«, ein Sextolenlauf im HexachordRahmen.

Besondere Bedeutung hat bei der Themenbildung die klassische Periode. Fast alle Themen, auch jene der späteren Fantasien, sind periodisch gebaut, satzartige Entwicklungstypen und alternative Themenaufbauten bilden die absolute Ausnahme.

Exempel 38a (Notenbeispiel 6) zeigt diesen Typus und übernimmt die kontrastierende Gestaltung der Segmente wie bereits im ausgehenden I8. Jahrhundert üblich, etwa in Mozarts Sonate c-Moll Kv 457 oder der Sinfonie C-Dur KV55I.

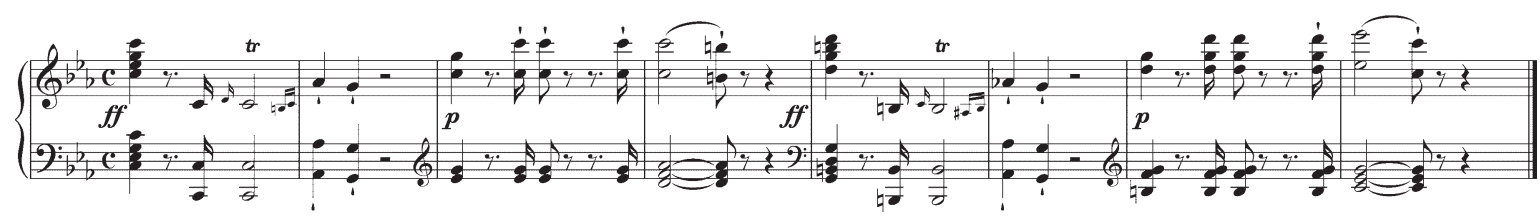

Notenbeispiel 6 Periodische Themengestaltung in Czernys op. 200, Exempel 38a, S. 37

Die Bevorzugung der Periodenstruktur lässt sich möglicherweise aus dem improvisatorischen Kontext erklären: Mit der symmetrischen Ordnung lässt sich die Gestaltung zweier gegensätzliche Motive sehr gut motivisch lösen, die klare Kadenzordnung gibt 
Orientierung, um die Gestalt leicht in der Erinnerung zu halten und im weiteren Verlauf in Variationen erneut zu verwenden. In den Modellkompositionen bringt Czerny die thematische Gestalt sogleich noch einmal in veränderter Satzstruktur. Auch Variationssätze, wie sie in den späteren Kapiteln erfolgen, sind in gefestigter Periodizität und klarer Viertakt-Symmetrie leichter zu gestalten.

Das Entwicklungsdenken des »Satz«-Typus (nach Erwin Ratz) erfordert ein höheres $\mathrm{Maß}$ an kompositorischer Planung, geht der Nachsatz doch meist von einer komplexen Verdichtung der Anfangsmotivik aus, die nicht selten von übergeordneten diastematischen Zügen oder Spiegelungen et cetera geleitet ist. Nur selten und in seiner einfachsten Form, der standardisierten Sequenzierung eines Motivs, tritt er in Czernys Themenbildungen auf, so etwa im Hauptthema des Allegro con brio der ersten Beispielfantasie Exempel 42: Nach einem periodischen Vordersatz wird das einfache Akkordschlagmotiv des Anfangstaktes repetiert und in den Halbschluss geführt (die Halbschlussbildung zu Beginn von Beethovens 5. Sinfonie (Takt 2I) mag hier Pate gestanden haben, sowohl was die harmonischen Gestaltung mittels des übermäßigen Sextakkordes betrifft als auch in der drastischen Gestik der Schlusswirkung mit Fermate.

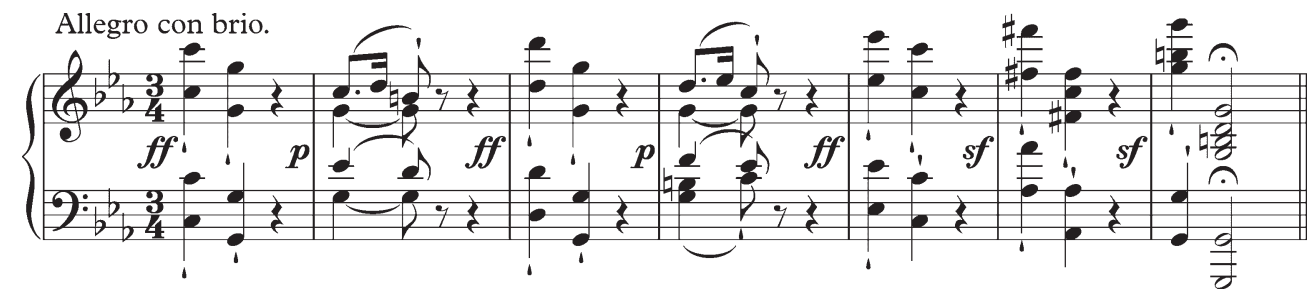

NOTEN B E I S P I L 7 Siebentaktiger »Satz《 mit periodischem Vordersatz in Czernys op. 200, Exempel 42 (Takt 13-19), S. 44

Für den weiteren Verlauf spricht Czerny die Notwendigkeit motivbasierter Verarbeitungstechniken als Zusammenhangsstiftung an.55 »Auffallende Noten« müssten »als Hauptfigur durch das Ganze, nach allen Imitationsregeln« eingewoben werden, wie Notenbeispiel 8 zeigt.

Diese thematischen Formungen werden in Einheiten von Taktgruppen integriert. Anhand der ersten Beispielfantasie Exempel 42 soll dieses Prinzip der Phrasengestaltung gezeigt werden. Dabei sind klare Strategien zu erkennen, um geschlossene Takteinheiten

Selbstverständlich ist der Begriff der motivischen beziehungsweise thematischen Arbeit zu Czernys Zeit noch nicht in Gebrauch, ebenso wenig verwendet er den Motivbegriff. Vgl. zu Verwendung und Geschichte der Begriffe: Christoph von Blumröder: Thematische Arbeit, motivische Arbeit, in: Handwörterbuch der musikalischen Terminologie I9 (HmT), hg. von Hans-Heinrich Eggebrecht, Freiburg i.Br. I99I, sowie: Motivisch-thematische Arbeit als Inbegriff der Musik? Zur Geschichte und Problematik eines >deutschen $<$ Musikdiskurses, hg. von Stefan Keym, Hildesheim u. a. 2015. 


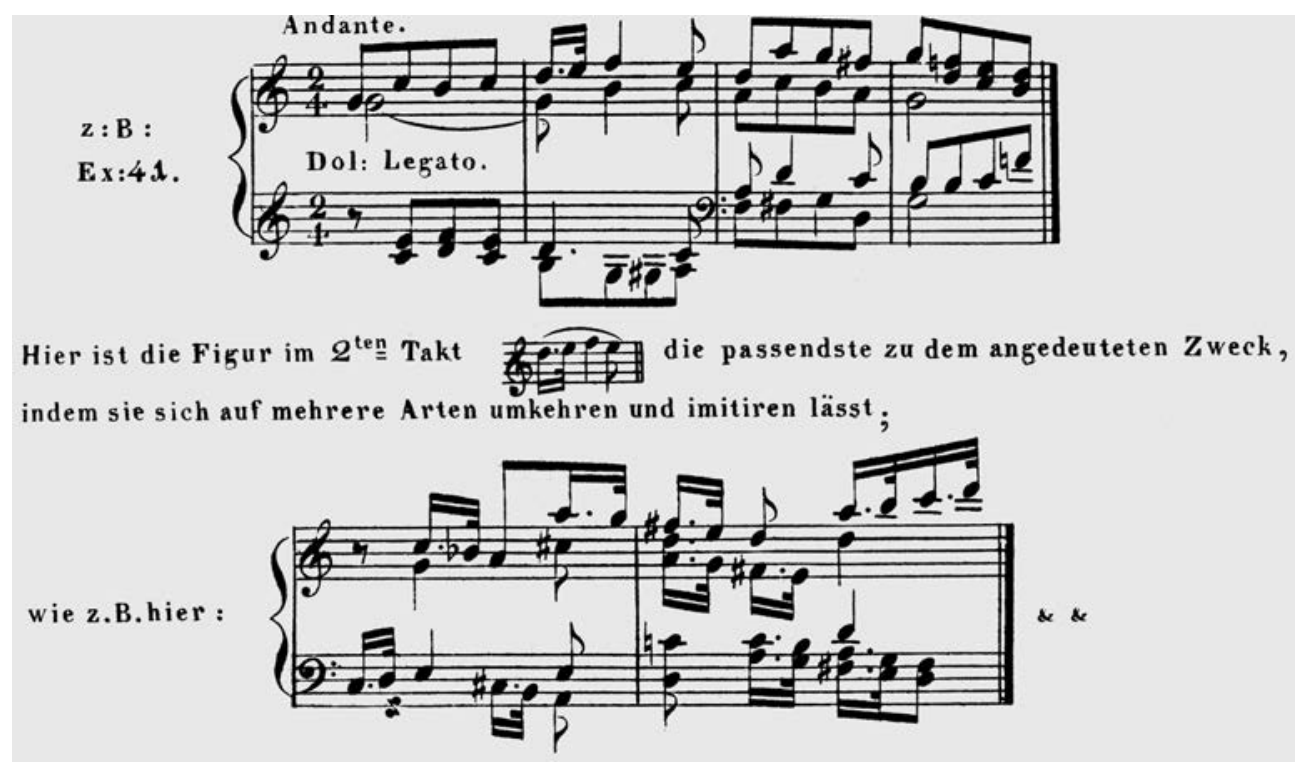

NOtENBEISPIEL 8 Motivische Verdichtung in Czernys op. 200, S. 42

von zumeist acht Takten improvisatorisch, aber doch in kohärentem Zusammenhang zu bilden:

Dem gesamten Verlauf liegt im Grunde eine Abfolge erlernter Muster, Bassverläufe und Sequenzen zugrunde. Anhand des Beginns des Allegro con brio soll dies exemplarisch gezeigt sein, Notenbeispiel 9 zeigt den Fortgang nach dem Hauptthema in c-Moll (Notenbeispiel 7): Es wird zunächst wiederholt(Segment b) und dabei in eine stabile Kadenz geführt, sodass sich insgesamt abermals eine periodische Formulierung des Themas ergibt. Die Bassfolge übernimmt den T-D/D-T-Chiasmus des Anfangs, um über die

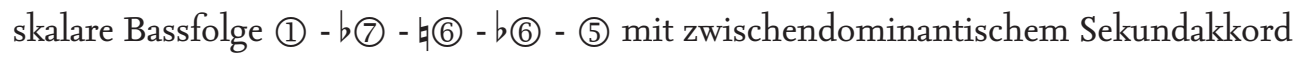
auf der 7. Bassstufe in die Kadenz zu gelangen. Die folgende Achtelrepetition gibt einen Takt lang die Gelegenheit, eine neue Entwicklung anzugehen, gewissermaßen durchzuatmen. Es folgt abermals ein periodischer Satz (Segment c), der über eines der häufigsten Muster in die parallele Durtonart Es-Dur moduliert: die grundständig harmonisierte Bassfolge $c-b-e s\left(c-B^{b}-E s\right)$ wird durch den zusätzlichen Basston ces und den darauf erklingenden übermäßigen Sextakkord erweitert. ${ }^{5}$ Es folgt ein Fauxbourdon-Gang aufwärts (11 $5^{-6}$ - (2) $7^{-6}-\left(37^{7-6}\right.$ - (4) $7^{-6}$ - (5) - (6) ${ }^{\text {aug6 }}$ - (5) mit stereotyper Harmonisierung (Segment d), jetzt in der Zieltonart Es-Dur. Die erreichte Dominante könnte nun in das

56 Diese chromatische Erweiterung des aufsteigenden Romanesca-Sequenzmodells, sowohl isoliert als auch im sequenzierten Zusammenhang, findet sich im frühen I9. Jahrhundert häufig. Bei Schubert mehrfach, indem der zusätzliche Halbtonschritt durch Liegenlassen der Oberstimmen als übermäßiger Durchgangsakkord erklingt, etwa in der Ouvertüre zu Die Zauberharfe D 644, Takt I7 ff. 
zweite Formulierung des Themas, nun im Bass $\quad 2 \mathrm{~T}$.

b)

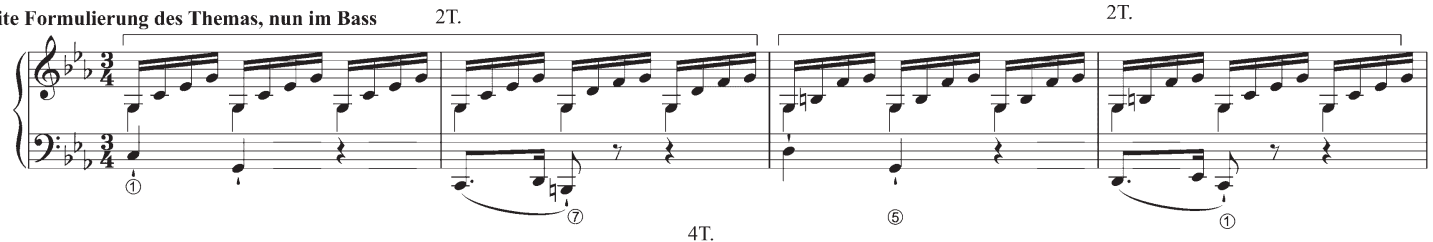

$4 \mathrm{~T}$

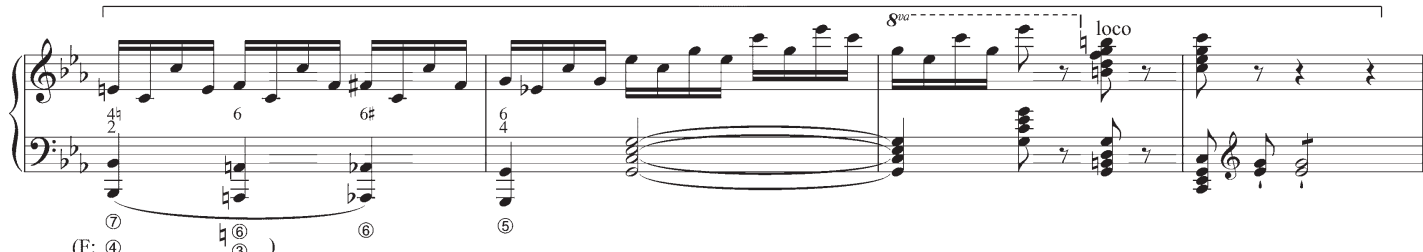

modulierende Periode

4T. (Vordersatz)

4T. (mod. Nachsatz)

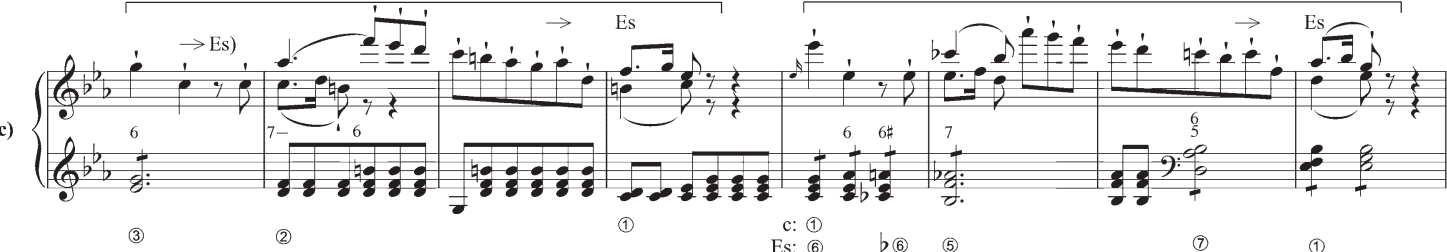

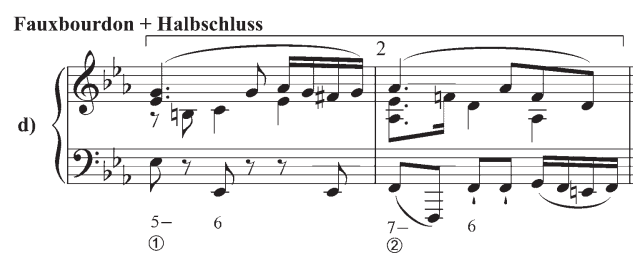

Es: (6) $\quad b_{(6)} \quad$ (5)

phryg. Halbschluss mit augm 6

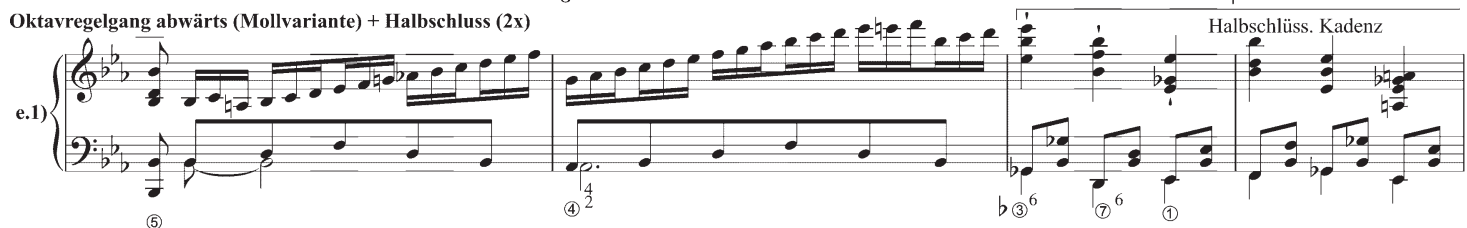

Halbschlüss. Kadenz

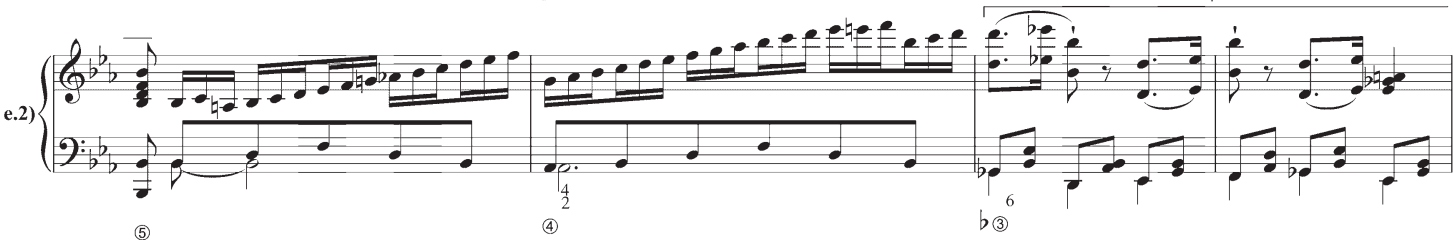

Oktavregel-Gang als Überleitung in den Seitensatz

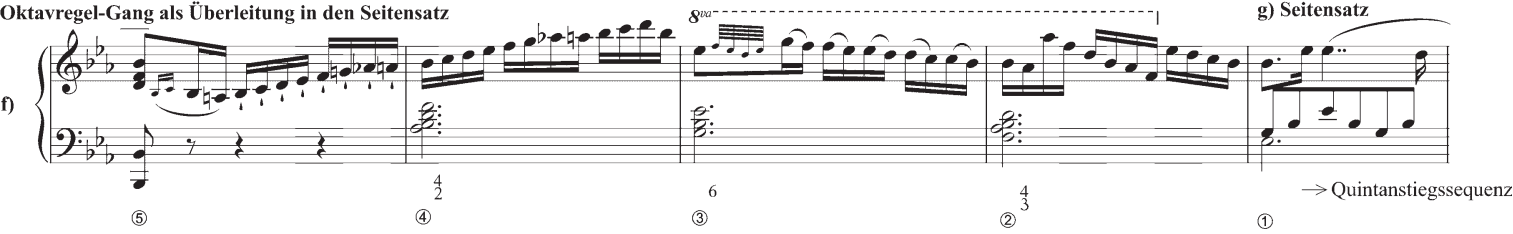

NOTENBEISPIEL 9 Phrasengestaltung nach normhaften Mustern in Czernys op. 200, Exempel 42 (Takt 19-53), S. 45 f. Aus Gründen der besseren Lesbarkeit ist die Passage ohne die zahlreichen Vortragsbezeichnungen zu Tempo und Dynamik wiedergegeben. 
Seitensatzthema führen, wird jedoch durch Passagenwerk verlängert, über OktavregelHarmonik (5) - (4) $)^{2}-b(3)^{6}-(7)^{6}$ - (1) und durch variantes es-Moll als temporärem Zielklang angereichert (Segment e, erscheint zweimal). Über den vollständigen Leiterabstieg von der 5. zur I. Bassstufe (Segment f, strikt oktavregelgemäß) wird schließlich das Thema des Seitensatzes (in Czernys Terminologie der »Mittelgesang«) erreicht, das aus einer einfachen Quintanstiegssequenz auf Basis des motivischen Grundmaterials besteht (Segment g).

Die Detailbetrachtung sei hier abgebrochen, aber sämtliche Beispiele ließen sich Phrase für Phrase nach diesem Verfahren beschreiben. Wichtig ist dabei festzuhalten: Nur durch dieses Abrufen gelernter Muster, die im Moment mit der Hauptmotivik und passenden, ebenfalls eingeübten Satzstrukturen kombiniert werden, ist eine Gestaltung solcher großformal gebundener Tonstücke möglich. Natürlich finden sich diese Formeln in ähnlicher Weise auch in komponierten Tonstücken, jedoch ist die Ausschließlichkeit der Anwendung als Baukastenprinzip bezeichnend. Improvisatorische Musik ist nicht nur durch das Moment der Überraschung, durch die Überschreitung des Normhaften in ungewohnten harmonischen Wendungen und tonalen Relationen gekennzeichnet - auch dieses findet sich bei Czerny, insbesondere in Überleitungspassagen, in den Präludien und im letzten Notenbeispiel des Capriccios -, sondern genauso vom scheinbaren Gegenteil: In Verdichtung lassen sich normhafte Strukturen und Klangverbindungen der Zeit ablesen, die sichere pianistische Verwendung einer oktavregelbasierten Harmonik ist dabei auch im I9. Jahrhundert unabdingbare Voraussetzung. Czerny selbst erwähnt sie nicht, aber die Mehrheit der harmonischen Verläufe folgt einem solchen Denken. Dass sie für das Erlernen die entscheidende Basis darstellt, zeigen nicht nur zahlreiche Beispiele vor Czerny, sondern auch danach: Friedrich Kalkbrenners Traité d'harmonie du Pianiste (I849), dezidiert als Improvisationslehre entworfen, behandelt in einem eigenen Kapitel die Oktavregel, sowohl in Gestalt der Lehrwerke des I8. Jahrhunderts als auch als zeitgenössische »nouvelle règle de l'octave« mit zusätzlichen verminderten Septakkorden und Zwischendominanten.57

Auch die Themengestaltungen sind bei Czerny davon betroffen. Notenbeispiel ro zeigt das Thema des langsamen Satzes Adagio espressivo von Exempel 42, dessen periodischer Vordersatz einem vollständigen Oktavregelverlauf abwärts folgt. Statt des Halbschlusses folgt eine ausweichende Kadenz in die II. Stufe, deren Offenheit nach vier Takten normhaft eingeholt wird. Auch dieses Kadenzverhältnis ist als Variante im frühen I9. Jahrhundert verbreitet, Schubert wendet es beispielsweise im langsamen Satz seiner

57 Friedrich Kalkbrenner: Traité d'harmonie du Pianiste. Principes rationnels de la modulation, pour apprendre à préluder et à improviser. Exemples d'Etudes, de Fugues et de Préludes pour le Piano, Paris/ Leipzig [I849], S.30 ff. 


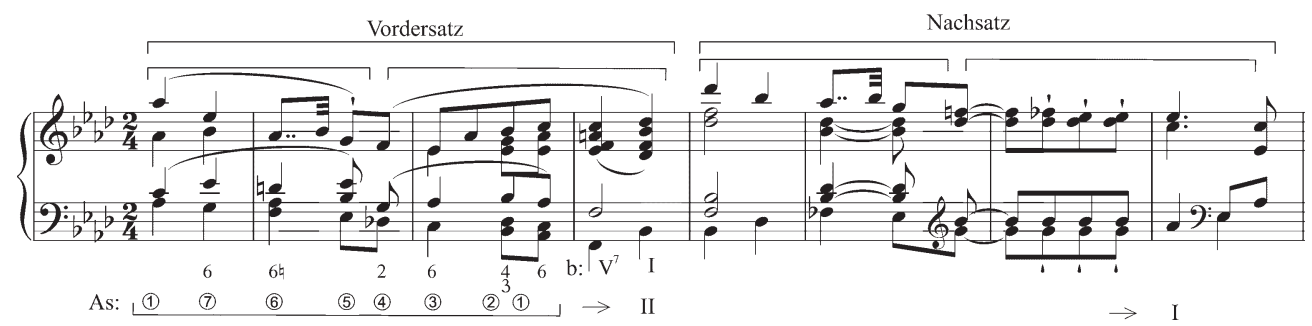

Noten BEISPIEL 10 Themenbildung nach Oktavregel in Czernys op. 200, Exempel 42, S. 48

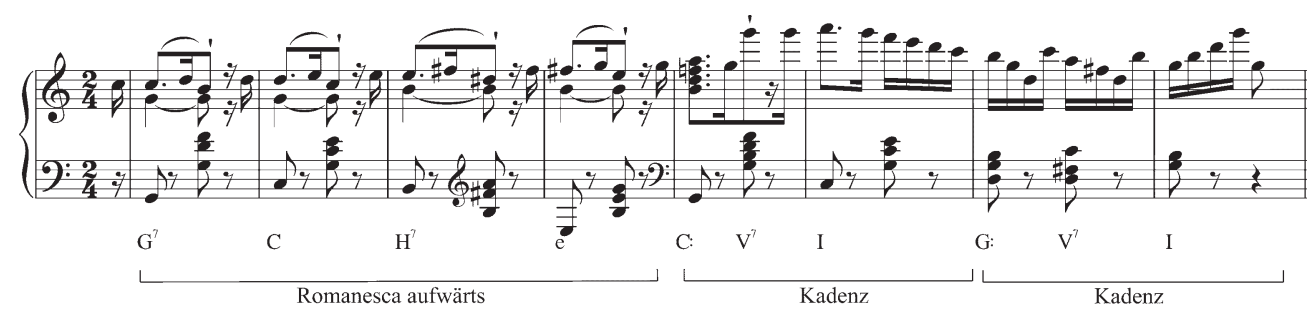

NOTEN BEISPIEL 11 Themenbildung nach Sequenzmustern in Czernys op. 200, Exempel 42, S. 50

5. Sinfonie B-Dur an (D 485), er selbst konnte bereits auf klassische Vorbilder zurückblicken.

Ebenso eignen sich Sequenzverläufe zur thematischen Gestaltung, insbesondere wenn diese zwar nicht in Originalgestalt aber doch wiedererkennbar nochmals erscheinen sollten. Dies ist in improvisierter Musik nur bedingt möglich, Czerny erwähnt es selbst, im Falle des Hauptthemas eines Rondos ist dies aber eine Grundbedingung der Form. Czerny gestaltet das abschließende Rondo-Thema als aufsteigendes RomanescaModell plus modulierende Kadenz in die Oberquinttonart (Notenbeispiel Ir). Die Formelhaftigkeit des Themas erlaubt ihm durch seine leichte Merkbarkeit die darauf Bezug nehmende Wiederkehr in anderen Satzstrukturen.

Improvisation wird in diesem Verständnis zu einem wenn auch extrem anspruchsvollen, so doch erlernbaren Handwerk, zu einem Komponieren im Moment. Einem Handwerk allerdings, das in seinem zeitintensiven Lernprozess schon bald nicht mehr den Übeanforderungen gerecht wird: Die Ausbildung einer hyper-virtuosen Technik und die Repertoireerweiterung zu einem schier unüberschaubaren Werkkorpus fordern ihren Tribut und erlauben Konzertpianisten des ausgehenden I9. Jahrhunderts diese schöpferische Freiheit nicht mehr, sie degradieren sie zu »Interpreten«. 


\section{Inhalt}

Vorwort 7

Maria Grazia Sità Improvisation and the Rhetoric of Beginning

Lutz Felbick Der Compositor extemporaneus Beethoven als »Enkelschüler« Johann Sebastian Bachs 34

Giorgio Sanguinetti A Partimento in Classical Sonata Form by Giacomo Tritto 57

Michael Lehner »Und nun sehe man, was hieraus gemacht werden kann«. Carl Czernys Anleitung zum Fantasieren als implizite Harmonie- und Formenlehre 69

Leonardo Miucci Completing the Score.

Beethoven and the Viennese Piano Concerto Tradition $\quad 9^{8}$

Martin Skamletz Joseph Preindls Klavierfantasien als Echo von Opern- und Oratorienaufführungen in Wien um I800 II6

Martin Skamletz »Classisches Clavierspiel«. Joseph Lipavsky und das Rondeau-Fantaisie $\quad$ I37

Sonja Wagenbichler Showdown am Klavier. Zur Kultur pianistischer Wettstreite im Wien des I8. und I9. Jahrhunderts $\quad$ I64

Stephan Zirwes Formale Dispositionen in den komponierten Fantasien zur Zeit Beethovens I75

Nathalie Meidhof Variation, »Harmoniekenntniss« und Improvisation. Beethovens Fünf Variationen über das englische Volkslied »Rule Britannia« für Klavier in D-Dur (WoO 79) I92

Namen-, Werk- und Ortsregister 202

Die Autorinnen und Autoren der Beiträge $\quad 208$ 


\section{DAS FLÜCHTIGE WERK}

Pianistische Improvisation der Beethoven-Zeit •

Herausgegeben von Michael Lehner, Nathalie Meidhof

und Leonardo Miucci unter redaktioneller

Mitarbeit von Daniel Allenbach 


\section{MUSIKFORSCHUNG DER \\ Hochschule Der KÜnste Bern \\ Herausgegeben von Martin Skamletz \\ und Thomas Gartmann}

Band 12 
0 Dieses Buch ist in gedruckter Form im Juli 20I9 in erster Auflage in der Edition Argus in Schliengen/Markgräflerland erschienen. Gestaltet und gesetzt wurde es im Verlag aus der Seria und der SeriaSans, die von Martin Majoor im Jahre 2000 gezeichnet wurden. Gedruckt wurde es auf Eos, einem holzfreien, säurefreien, chlorfreien und alterungsbeständigen Werkdruckpapier der Papierfabrik Salzer im niederösterreichischen Sankt Pölten. Das Vorsatzpapier Caribic cherry wurde von Igepa in Hamburg geliefert. Rives Tradition, ein Recyclingpapier mit leichter Filznarbung, das für den Bezug des Umschlags verwendet wurde, stellt die Papierfabrik Arjo Wiggins in Issyles-Moulineaux bei Paris her. Das Kapitalband mit rot-schwarzer Raupe lieferte die Firma Dr. Günther Kast aus Sonthofen im Oberallgäu, die auf technische Gewebe und Spezialfasererzeugnisse spezialisiert ist. Gedruckt und gebunden wurde das Buch von der Firma Bookstation im bayerischen Anzing. Im Internet finden Sie Informationen über das gesamte Verlagsprogramm unter www.editionargus.de, zum Institut Interpretation der Hochschule der Künste Bern unter www.hkb.bfh.ch/interpretation und www.hkb-interpretation.ch. Die Deutsche Nationalbibliothek verzeichnet diese Publikation in der Deutschen Nationalbibliografie; detaillierte bibliografische Daten sind im Internet über www.dnb.de abrufbar. (C) der zeitgleich erschienenen digitalen Version: die Autorinnen und Autoren, 2019. Dieses Werk ist lizenziert unter einer Creative Commons Namensnennung-Nicht kommerziell 4.0 International Lizenz (CC BY-NC 4.0). Dor: https://doi.org/I0.26045/kp64-6I76 ISBN 978-3-93I264-92-5 